\title{
An efficient algorithm to analyze wave propagation in fluid/solid and solid/fluid phononic crystals \\ Eric $\mathrm{Li}^{2}, \mathrm{ZC} \mathrm{He}^{1 *}$, Gang Wang ${ }^{1}$, GR $\mathrm{Liu}^{3}$ \\ ${ }^{1}$ State Key Laboratory of Advanced Design and Manufacturing for Vehicle Body, Hunan University, Changsha, 410082 P. R. China \\ ${ }^{2}$ Department of Mechanical and Automation Engineering, The Chinese University of Hong Kong, Shatin, NT, Hong Kong, China \\ ${ }^{3}$ Department of Aerospace Engineering and Engineering Mechanics, University of Cincinnati, Cincinnati, OH 45221-0070, USA
}

\begin{abstract}
The paper presents an efficient and accurate algorithm to analyze the fluid/solid and solid/fluid phononic crystals (PCs) with fluid structure interaction (FSI). In the proposed mass-redistributed finite element method (MR-FEM), the dispersion error in the longitudinal wave of fluid domain is minimized by re-distributing the mass elements in the mass matrix to "tune" the balance, while the lumped mass matrix is employed to analyze the elastic wave within the solid. In addition, different Bloch boundary conditions including the square and triangular lattice using MR-FEM are also formulated in this work. The accuracy of the proposed MR-FEM is verified by using the commercial software COMSOL, and the computational efficiency of MR-FEM is significantly greater than the conventional finite element methods (FEM) based on six different numerical examples. The MR-FEM with excellent features has a great potential to extend 3D multiphysics domain of PCs.
\end{abstract}

Key words: Phononic Crystals; Fluid Structure Interaction; Band Gap; Mass-redistributed Finite Element Method (MR-FEM)

\footnotetext{
1* State Key Laboratory of Advanced Design and Manufacturing for Vehicle Body, Hunan University, Changsha, 410082 P. R. China Email address: hezhicheng815@hnu.edu.cn
} 


\section{Introduction}

The scientific research of phononic crystals (PCs) has gained an increasing amount of attention due to their remarkable and useful physical properties which are not found in nature [1-6]. Different from traditional materials, the mechanical properties of PCs are related to the periodic arrangement of two or more materials in representative volume element (RVE) instead of chemical constituents [7-9]. In 1968, Veselago firstly proposed a theoretical possibility to develop a material with simultaneously negative electric permittivity and magnetic permeability [10], which is also termed as left hand materials (LFM) [11]. Three decades later, this concept has become a reality with experimental verification by Shelby et al. who designed structure metamaterials with copper strips and split ring resonators in 2001[12].

Driven by the similar concept between electromagnetic and acoustic waves, the development of counterpart PCs has grown tremendously in the last decade [13-15]. The unusual and useful mechanical properties of PCs with manmade periodic microstructures structure have been explored to design noise and vibration isolators, acoustic waveguides and mechanical filters $[13,16,17]$. In particular, the existence of band gap in the PCs to stop the propagation of waves of certain frequencies has attracted a lot of attention from researchers recently [18-23]. The past studies have indicated that there are two working mechanisms of band gap in PCs, which is known as Bragg scattering and local resonance $[24,25]$. In general, the propagating wavelength must be on the order of the microstructure caused by the Bragg scattering in order to create the band gaps [25]. Therefore, gigantic engineering structures are needed for vibration shielding in the low 
frequency range [24], which has become a bottleneck in the advanced development of PCs based on Bragg scattering.

In 2000, Liu et al. first discovered local resonance of sonic materials (LRSMs) by using the lead balls coated with silicone rubber [26], which has demonstrated that the size of microstructures is much smaller than the wavelength of the wave in epoxy. Since then, numerous works and efforts have been made to improve the band gaps in PCs [27]. Li and Chan have demonstrated theoretically the concept of a double-negative PCs with negative effective bulk modulus and density in 2004 [28]. Liu et al. proposed a broadband elastic metamaterial with single negativity by mimicking lattice systems [29]. Except for passive PCs, the tuning of band gaps is essential to applications of PCs. Chen et al. first investigated the band gap control of an active mass-in-mass lattice system [30]. Furthermore, Liu et al. have reviewed the micromachined tunable metamaterials based on the mechanical reconfiguration of the lattice and the element geometry [8].

In parallel with theoretical studies, the development of efficient, robust and accurate numerical methods is very important to investigate the wave motion in various types of PCs with multi-physics domains [31-39]. At the early design stages, the plane-wave expansion (PWE) [40, 41] and finite difference method (FDM) [42] were widely used to model the physical response of PCs. However, the PWE and FDM have some difficulties in the handling fluid-solid interface conditions [43]. In 2006, a wavelet-based method was developed by Yan and Wang to calculate the elastic band gaps of 2D PCs, which can reduce the Gibbs effect to a certain extent [44]. On the other hand, finite element method (FEM) with easy treatment of complex boundary and interface is becoming more and more popular in the simulation of PCs. Li et al. used the commercial software ABAQUS 
to compute and analyze the band gaps of 2D and 3D PCs [45, 46]. In 2007, a fluid-solid coupling numerical algorithm was proposed by Liu and Gao based on explicit dynamic finite element method (DFEM) to compute the band-structure [47]. Consequently, Li et al. [48] have used a boundary element method (BEM) to compute the band structures of mixed solid - fluid phononic crystals. Shi et al. developed a generalized multipole technique to solve the systems with fluid inclusions in solids or with solid inclusions in fluid matrix $[49,50]$. Recently, a meshfree or meshless local radial basis function (RBF) collocation method was developed to compute the band structures of two-dimensional anti-plane transverse elastic waves in PCs $[43,51]$.

In the simulation of fluid/solid and solid/fluid PCs with FSI, mixed pressure and shear waves propagate in solids, while only longitudinal wave exists in ideal fluids [48]. Our past research has found that the dispersion error of longitudinal wave in the fluid domain is mainly caused by the balance between the stiffness and mass [52, 53]. The theoretical studies and numerical examples have shown that the balance between the stiffness and mass matrices for longitudinal wave can be controlled by 1) softening the overly-stiff traditional finite element model; or 2) adjusting the mass matrix with respect to the stiffness of the system. In the past, many efforts have been made to soften the overly-stiff of traditional FEM model, especially in the area of smoothed finite element methods (S-FEM) [54-57]. However, the extra computational effort to form the smoothed stiffness and large bandwidth of system matrix are of great burden in the process of large scale problems. The re-distribution of the entries in the mass matrix is able to improve the balance of the stiffness and mass of a discretized model, which reduces the dispersion error significantly [58]. This efficient mass-redistributed finite element method (MR- 
FEM) that is lured by modified integration rule [59, 60], has been applied to vibroacoustic interaction and heat transfer problems successfully [53, 61, 62]. With employment of simple triangular elements, such balance between the stiffness and mass can be easily done by shifting the integration points in the evaluation of mass matrix. The implementation of MR-FEM is very straightforward without changing standard FEM code too much. Hence, the computational cost almost remains unchanged compared with traditional FEM.

Motivated from the excellent properties of MR-FEM to minimize the dispersion error in the simulation of longitudinal wave, this work formulated a system to tune the balance between the mass and stiffness, which is used to analyze the physical response of PCs with FSI. In the elastic domain, the lumped mass matrix is employed to model the propagation of elastic wave [63]. In the fluid part, the MR-FEM is adopted to find the optimal integration point to minimize the dispersion error of longitudinal wave. The theoretical analysis and numerical examples have demonstrated that the present MR-FEM works ideally for fluid/solid and solid/fluid PCs with FSI: it can significantly reduce the numerical dispersion error from low to mid frequency ranges compared with the standard FEM using the same triangular mesh. The proposed MR-FEM with its distinct features is unique and innovative, which has a strong potential in the design and analysis of PCs for 3D large scale problems. The purpose of this paper is to establish an accurate, robust and stable algorithm to analyze the wave propagation in PCs with FSI, which provides an efficient tool to design PCs with multifunction.

The rest of paper is structured as follows: Section 2 describes the mathematical model of fluid/solid and solid/fluid PCs with FSI. The optimal integration points in the 
MR-FEM based on the dispersion error analysis are given in Section 3. The accuracy and computational efficiency of MR-FEM in the analysis of wave propagation within fluid/solid and solid/fluid PCs with different Bloch boundary conditions are presented in Section 4. Finally, the discussions and conclusions of current work are made in Section 5.

\section{Mathematical model of fluid/solid and solid/ fluid PCs}

\subsection{Governing equation of wave motion in PCs with FSI}

As outlined in Fig. 1, a typical representation of 2D periodic PCs with FSI is presented. According to the theory of Bloch-theorem, only one unit-cell is considered. The interface between the solid and fluid is shown in Fig. 1. If the solid media is considered as isotropic material, the governing equation for the motion of the elastic wave is given by:

$$
\begin{aligned}
& \mu \frac{\partial^{2} u_{x}}{\partial y^{2}}+(\lambda+2 \mu) \frac{\partial^{2} u_{x}}{\partial x^{2}}+(\lambda+\mu) \frac{\partial^{2} u_{y}}{\partial x \partial y}=\rho \ddot{u}_{x} \\
& \mu \frac{\partial^{2} u_{y}}{\partial x^{2}}+(\lambda+2 \mu) \frac{\partial^{2} u_{y}}{\partial y^{2}}+(\lambda+\mu) \frac{\partial^{2} u_{x}}{\partial x \partial y}=\rho \ddot{u}_{y}
\end{aligned}
$$

where $u_{x}$ and $u_{y}$ are the induced displacements along $x$ and $y$ directions, respectively, and $\rho$ is the density. In the $2 \mathrm{D}$ problem, $\lambda$ and $\mu$ denote the Lamé's first parameter and shear modulus.

$$
\mu=\frac{E}{2(1+v)}, \lambda=\frac{v E}{(1+v)(1-2 v)}
$$

where $E$ and $v$ represent the Young's modulus and Poisson's ratio, respectively.

The constitutive relationship for isotropic linear elasticity is expressed as follows:

$$
\boldsymbol{\sigma}=\mathbf{D} \boldsymbol{\varepsilon}
$$


where $\mathbf{D}$ is a symmetric positive definite (SPD) matrix of material related to Poisson's ratio and Young's modulus, $\boldsymbol{\sigma}$ is the stress, and $\boldsymbol{\varepsilon}$ is the strain. The matrix of material constants $\mathbf{D}$ can be expressed as follows:

$$
\mathbf{D}=\left[\begin{array}{ccc}
\lambda+2 \mu & \lambda & 0 \\
\lambda & \lambda+2 \mu & 0 \\
0 & 0 & \mu
\end{array}\right]
$$

The strain is given by:

$$
\varepsilon_{i j}=\frac{1}{2}\left(\frac{\partial u_{i}}{\partial x_{j}}+\frac{\partial u_{j}}{\partial x_{i}}\right) \quad i, j=1,2
$$

In the frequency domain, the propagation of elastic wave in Eq. (1) can be expressed in the following form [64]:

$$
\mu \nabla^{2} \mathbf{u}+(\lambda+\mu) \nabla(\nabla \cdot \mathbf{u})=-\rho \omega^{2} \mathbf{u}
$$

where $\omega$ is the angular frequency of mechanical motion.

In the fluid part, the governing equation of longitudinal wave is expressed as follows:

$$
\Delta p^{\prime}-\frac{1}{c^{2}} \frac{\partial^{2} p^{\prime}}{\partial t^{2}}=0 \text { in } \Omega
$$

where $\Delta$ is the Laplace operator, $c$ and $t$ are the velocity of sound propagating in the medium and time, respectively. Here the acoustic pressure $p^{\prime}$ is a small harmonic perturbation around the steady state with the form given as follows:

$$
p=p e^{j \omega t}
$$

where $p$ is the spatial distribution of complex acoustic pressure and satisfies the following Helmholtz Equation [56]:

$$
\Delta p+k^{2} p=0
$$

where $k$ is the wavenumber defined by 


$$
k=\frac{\omega}{c}
$$

In the propagation of wave within fluid/solid and solid/fluid PCs as shown in Fig. 1 (the symbol A or B can be either fluid or solid in Fig. 1), the structural domain $\Omega_{s}$ is coupled with the fluid domain $\Omega_{f}$ at the interface. During the interaction between the acoustic and elastic waves, the solid and fluid particles move together in the normal direction of the interface.

$$
u_{s} \mathbf{n}=u_{f} \mathbf{n} \quad \text { on } \Gamma_{s f}
$$

where $u_{s}$ and $u_{f}$ represent the displacements of elastic and fluid particles at the interface, respectively. The $\mathbf{n}$ is the normal vector along the interface. At the interface, the continuity between the structural stress and acoustic pressure can be expressed as follows:

$$
\begin{gathered}
\tau_{x}=\sigma_{x x} n_{x}+\sigma_{x y} n_{y}, \tau_{y}=\sigma_{y y} n_{x}+\sigma_{x y} n_{x} \\
\tau_{x}=p n_{x}, \tau_{y}=p n_{y}
\end{gathered}
$$

where $\tau$ is the traction force.

The fluid force exerting on the structure along the interface can be computed:

$$
\mathbf{F}_{s}=\int_{\Gamma_{s f}} \mathbf{N}_{s}^{\mathrm{T}} \tau d \Gamma=\int_{\Gamma_{s f}} \mathbf{N}_{s}^{\mathrm{T}} \mathbf{n} p d \Gamma=\left(\int_{\Gamma_{s f}} \mathbf{N}_{s}^{\mathrm{T}} \mathbf{n} \mathbf{N}_{f} d \Gamma\right) \mathbf{p}
$$

where $\mathbf{N}_{s}$ and $\mathbf{N}_{f}$ are the shape functions in the solid and fluid domains.

Analogously, the force from the structure acting on the fluid can be derived:

$$
\mathbf{F}_{f}=-\rho_{f} \int_{\Gamma_{s f}} \mathbf{N}_{f}^{\mathrm{T}} \ddot{u_{f}} d \Gamma
$$

By introducing the spatial coupling matrix: 


$$
\mathbf{H}=\int_{\Gamma_{s f}} \mathbf{N}_{s} \mathbf{n}_{f} \mathbf{N}_{f} \mathrm{~d} \Gamma
$$

The coupling forces in Eqs. (15) and (16) can be written as

$$
\mathbf{F}_{s}=\mathbf{H p}, \quad \mathbf{F}_{f}=-\rho \mathbf{H}^{T} \ddot{\mathbf{u}}_{s}
$$

Based on Eqs. (15) and (16), the fluid/solid or solid/fluid PCs with FSI can then be described by the following unsymmetrical equations:

$$
\left[\begin{array}{cc}
\mathbf{M}_{s} & 0 \\
\rho \mathbf{H}^{T} & \mathbf{M}_{f}
\end{array}\right]\left[\begin{array}{c}
\ddot{\mathbf{u}}_{s} \\
\ddot{\mathbf{p}}
\end{array}\right]+\left[\begin{array}{cc}
\mathbf{K}_{s} & -\mathbf{H} \\
0 & \mathbf{K}_{f}
\end{array}\right]\left[\begin{array}{l}
\mathbf{u} \\
\mathbf{p}
\end{array}\right]=\left[\begin{array}{c}
\mathbf{F}_{s} \\
\mathbf{F}_{f}
\end{array}\right]
$$

Assume the pressure and displacement are both time harmonic, the force frequency response analysis equation for fluid/solid and solid/fluid PCs can be formulated as follows:

$$
\left[\begin{array}{cc}
\mathbf{K}_{s}-\omega^{2} \mathbf{M}_{s} & -\mathbf{H} \\
\rho_{f} \omega^{2} \mathbf{H}^{T} & \mathbf{K}_{f}-\omega^{2} \mathbf{M}_{f}
\end{array}\right]\left[\begin{array}{l}
\mathbf{u} \\
\mathbf{p}
\end{array}\right]=\left[\begin{array}{c}
\mathbf{F}_{s} \\
\mathbf{F}_{f}
\end{array}\right]
$$

Using standard Galerkin procedure with triangular element, the stiffness, mass and force matrices in Eq. (20) are written as follows:

$$
\begin{array}{cr}
\mathbf{K}_{s}=\int_{\Omega} \mathbf{B}_{\mathrm{s}}^{\mathrm{T}} \mathbf{D} \nabla \mathbf{B}_{\mathrm{s}} \mathrm{d} \Omega & \text { The stiffness matrix for elastic domain } \\
\mathbf{M}_{s}=\rho_{s} \int_{\Omega} \mathbf{N}_{\mathrm{s}}^{\mathrm{T}} \mathbf{N}_{\mathrm{s}} \mathrm{d} \Omega & \text { The elastic mass matrix } \\
\mathbf{K}_{f}=\int_{\Omega} \mathbf{B}_{f}^{\mathrm{T}} \mathbf{B}_{f} \mathrm{~d} \Omega & \text { The stiffness matrix for the fluid domain } \\
\mathbf{M}_{f}=\rho_{f} \int_{\Omega} \mathbf{N}_{f}^{\mathrm{T}} \mathbf{N}_{f} \mathrm{~d} \Omega & \text { The fluid mass matrix }
\end{array}
$$


where $\mathbf{B}_{s}$ is the strain matrix in the elastic domain, and $\mathbf{B}_{f}$ is the pressure gradient in the fluid domain.

\subsection{Treatment of boundary condition}

In this work, two types of lattice forms are discussed including square and triangular cells in fluid/solid and solid/fluid PCs. The displacement and pressure should satisfy the following Bloch theorem.

\subsubsection{Fluid/solid PCs}

In the fluid/solid PCs with square lattice shown in Fig. 1, only the elastic wave field of the solid matrix is applied at the boundary, where the displacements in the solid domain are expressed as follows [43, 48, 65]:

$$
\begin{aligned}
& \mathbf{u}_{x}\left(\mathbf{x}_{\Gamma_{1}}\right)=\mathbf{u}_{x}\left(\mathbf{x}_{\Gamma_{3}}\right) e^{-i k_{x} a}, \mathbf{u}_{y}\left(\mathbf{x}_{\Gamma_{1}}\right)=\mathbf{u}_{y}\left(\mathbf{x}_{\Gamma_{3}}\right) e^{-i k_{x} a} \\
& \mathbf{u}_{x}\left(\mathbf{x}_{\Gamma_{2}}\right)=\mathbf{u}_{x}\left(\mathbf{x}_{\Gamma_{4}}\right) e^{-i k_{y} a}, \mathbf{u}_{y}\left(\mathbf{x}_{\Gamma_{2}}\right)=\mathbf{u}_{y}\left(\mathbf{x}_{\Gamma_{4}}\right) e^{-i k_{y} a}
\end{aligned}
$$

In the fluid/solid PCs with triangular lattice shown in Fig. 1, the periodic boundary conditions of the displacements for the hexagonal cell are expressed as follows [43, 48, 65]:

$$
\begin{aligned}
& \mathbf{u}_{x}\left(\mathbf{x}_{\Gamma_{1}}\right)=\mathbf{u}_{x}\left(\mathbf{x}_{\Gamma_{4}}\right) e^{-i\left(k_{x} \frac{\sqrt{3}}{2} a+k_{y} \frac{1}{2} a\right)}, \mathbf{u}_{y}\left(\mathbf{x}_{\Gamma_{1}}\right)=\mathbf{u}_{y}\left(\mathbf{x}_{\Gamma_{4}}\right) e^{-i\left(k_{x} \frac{\sqrt{3}}{2} a+k_{y} \frac{1}{2} a\right)} \\
& \mathbf{u}_{x}\left(\mathbf{x}_{\Gamma_{2}}\right)=\mathbf{u}_{x}\left(\mathbf{x}_{\Gamma_{5}}\right) e^{-i k_{y} a}, \quad \mathbf{u}_{y}\left(\mathbf{x}_{\Gamma_{2}}\right)=\mathbf{u}_{x}\left(\mathbf{x}_{\Gamma_{5}}\right) e^{-i k_{y} a} \\
& \mathbf{u}_{x}\left(\mathbf{x}_{\Gamma_{3}}\right)=\mathbf{u}_{x}\left(\mathbf{x}_{\Gamma_{6}}\right) e^{i\left(k_{x} \frac{\sqrt{3}}{2} a-k_{y} \frac{1}{2} a\right)}, \mathbf{u}_{y}\left(\mathbf{x}_{\Gamma_{3}}\right)=\mathbf{u}_{y}\left(\mathbf{x}_{\Gamma_{6}}\right) e^{i\left(k_{x} \frac{\sqrt{3}}{2} a-k_{y} \frac{1}{2} a\right)}
\end{aligned}
$$

where $k_{x}$ and $k_{y}$ are real Bloch wave along $x$ and $y$ directions. 


\subsubsection{Solid/fluid PCs}

In the solid/fluid PCs, only the acoustic wave is applied to the periodic boundary. For a square lattice shown in Fig. 1, the pressure should satisfy the following equation [43, 48, 65]:

$$
\mathbf{p}\left(\mathbf{x}_{\Gamma_{1}}\right)=\mathbf{p}\left(\mathbf{x}_{\Gamma 3}\right) e^{-i k_{x} a}, \quad \mathbf{p}\left(\mathbf{x}_{\Gamma_{2}}\right)=\mathbf{p}\left(\mathbf{x}_{\Gamma_{4}}\right) e^{-i k_{y} a}
$$

Similarly, the periodic boundary conditions of the pressure for the triangular lattice shown in Fig. 1 are expressed as follows:

$$
\begin{gathered}
\mathbf{p}\left(\mathbf{x}_{\Gamma_{1}}\right)=\mathbf{p}\left(\mathbf{x}_{\Gamma_{4}}\right) e^{-i\left(k_{x} \frac{\sqrt{3} a}{2}+k_{y} \frac{a}{2}\right)} \\
\mathbf{p}\left(\mathbf{x}_{\Gamma_{2}}\right)=\mathbf{p}\left(\mathbf{x}_{\Gamma_{5}}\right) e^{-i k_{y} a} \\
\mathbf{p}\left(\mathbf{x}_{\Gamma_{3}}\right)=\mathbf{p}\left(\mathbf{x}_{\Gamma_{6}}\right) e^{i\left(k_{x} \frac{\sqrt{3} a}{2}-k_{y} \frac{a}{2}\right)}
\end{gathered}
$$

\section{Analysis of dispersion error of longitudinal wave in the liquid domain}

The main objective of MR-FEM is to propose three flexible integration points in the mass matrix, which aims to balance the stiffness and mass to minimize the dispersion error in the propagation of longitudinal wave. Different from traditional Gauss integration points, the following new locations of integration points as shown in Fig. 2 for the general mass matrix are proposed:

$$
\begin{gathered}
\text { Point } \alpha: \xi_{i}=\frac{1-q}{2}, \quad \eta_{i}=\frac{1-q}{2} ; \quad \text { Point } \beta: \xi_{i}=q, \quad \eta_{i}=\frac{1-q}{2} \\
\text { Point } \gamma: \xi_{i}=\frac{1-q}{2}, \quad \eta_{i}=q
\end{gathered}
$$

where $q \in[0,1]$.

Based on Eq. (31), the general mass matrix in the solid domain can be derived with a 
parameter $q$ :

$$
\mathbf{M}_{s}=\frac{1}{3} \rho_{s} A^{e}\left[\begin{array}{cccccc}
c_{11} & 0 & c_{13} & 0 & c_{15} & 0 \\
0 & c_{22} & 0 & c_{24} & 0 & c_{26} \\
c_{31} & 0 & c_{33} & 0 & c_{35} & 0 \\
0 & c_{42} & 0 & c_{44} & 0 & c_{46} \\
c_{51} & 0 & c_{53} & 0 & c_{55} & 0 \\
0 & c_{62} & 0 & c_{64} & 0 & c_{66}
\end{array}\right]
$$

where $A^{e}$ is the area of each triangular element. The parameters $c_{i j}$ are given as follows:

$$
\begin{gathered}
c_{11}=c_{22}=c_{33}=c_{44}=c_{55}=c_{66}=q^{2}+\frac{(1-q)^{2}}{2} \\
c_{13}=c_{15}=c_{24}=c_{26}=c_{31}=c_{35}=c_{42}=c_{46}=c_{51}=c_{53}=c_{62}=c_{64}=q(1-q)+\frac{(1-q)^{2}}{4}
\end{gathered}
$$

Analogously, the mass matrix in the fluid domain can be derived in the same way:

$$
\mathbf{M}_{f}=\frac{1}{3} \rho_{f} A^{e}\left[\begin{array}{ccc}
q^{2}+\frac{(1-q)^{2}}{2} & q(1-q)+\frac{(1-q)^{2}}{4} & q(1-q)+\frac{(1-q)^{2}}{4} \\
q(1-q)+\frac{(1-q)^{2}}{4} & q^{2}+\frac{(1-q)^{2}}{2} & q(1-q)+\frac{(1-q)^{2}}{4} \\
q(1-q)+\frac{(1-q)^{2}}{4} & q(1-q)+\frac{(1-q)^{2}}{4} & q^{2}+\frac{(1-q)^{2}}{2}
\end{array}\right]
$$

In the propagation of stress wave within the fluid/solid or solid/fluid PCs, the elastic wave in the solid domain can be decomposed into two parts:

$$
\mathbf{u}=\nabla \Phi+\nabla \times \boldsymbol{\psi}
$$

Put Eq. (7)

$$
\nabla\left[(\lambda+2 \mu) \nabla^{2} \Phi-\rho \ddot{\Phi}\right]+\nabla \times\left[\mu \nabla^{2} \boldsymbol{\psi}-\rho \ddot{\boldsymbol{\psi}}\right]=0
$$

The vector potential $\psi$ and scalar potential $\Phi$ can be further expressed in terms of Helmholtz equation. 


$$
\begin{array}{ll}
\nabla^{2} \Phi=\frac{1}{c_{p}^{2}} \frac{\partial^{2} \Phi}{\partial t^{2}} & \text { Pressure wave } \\
\nabla^{2} \boldsymbol{\psi}=\frac{1}{c_{s}^{2}} \frac{\partial^{2} \boldsymbol{\psi}}{\partial t^{2}} & \text { Shear wave }
\end{array}
$$

where

$$
c_{p}^{2}=\frac{\lambda+2 \mu}{\rho} \quad c_{s}^{2}=\frac{\mu}{\rho}
$$

The pressure and the shear stress wave lengths are given as follows:

$$
\chi_{p}=\frac{1}{f} \sqrt{\frac{E(1-v)}{\rho(1-2 v)(1+v)}} \quad \chi_{s}=\frac{1}{f} \sqrt{\frac{E}{2 \rho(1+v)}}
$$

where $f$ is the frequency of the wave.

The ratio between the two wave lengths can be expressed as

$$
\frac{\chi_{p}}{\gamma_{s}}=\sqrt{\frac{(1+v)}{(1-2 v)}}
$$

It is seen that the pressure and the shear stress waves are coupled with Poisson's ratio v. As shown in Fig. 3, the shear wave length is almost negligible as the Poisson's ratio is very close to 0.5 . In this work, the Poisson's ratio of solid is considered as compressible. Therefore, the effect of the availability of shear stress wave in solid domain cannot be ignored. Such coupling effects between the shear and pressure stress waves make it difficult to balance the discretized systems for the propagation of elastic wave within the solid domain using the same integration point simultaneously. Therefore, we only focus on how to balance the stiffness and mass in the fluid domain using MR-FEM, which aims 
to improve the computational efficiency of band gap of fluid/solid and solid/fluid PCs.

As shown in Fig. 4, a two dimensional problem is considered. The whole domain is discretized with a regular triangular mesh with a constant node spacing $d$ (or the shortest edge in the mesh). In Fig. 4, the nodes which contribute to the MR-FEM system equation involved with interior node $(i, j)$ are also labeled. The row of equation in the MR-FEM discretized system involves with the node $(i, j)$ which can be written as follows:

$$
\Gamma_{s}^{M R-F E M}=\eta_{1} p_{i, j+1}+\eta_{2} p_{i+1, j+1}+\eta_{1} p_{i-1, j}+\eta_{0} p_{i, j}+\eta_{1} p_{i+1, j}+\eta_{2} p_{i-1, j-1}+\eta_{1} p_{i, j-1}=0
$$

Here $\Gamma_{s}^{M R-F E M}$ stands for the row of discretized system equation involving with node ( $i$, $j$ ) in the MR-FEM.

The longitudinal wave in the fluid domain is expressed as follows:

$$
p(x)=P_{A} e^{i\left(k_{1} x+k_{2} y\right)} \text { with } k_{1}=k^{d} \cos \varphi \quad k_{2}=k^{d} \sin \varphi
$$

where $\varphi$ is the wave propagation angle.

The relation between the actual wavenumber $k$ and the approximate wavenumber $k^{d}$ can be obtained as

$$
k^{2}=\frac{1}{d^{2}} \frac{24-12\left[\cos \left(k_{1} d\right)+\cos \left(k_{2} d\right)\right]}{3\left(1-2 q+3 q^{2}\right)+\left(1+2 q-3 q^{2}\right)\left[\cos \left(k_{1} d\right)+\cos \left(k_{2} d\right)+\cos \left(k_{1} d+k_{2} d\right)\right]}
$$

Taking the Taylor expansion of $k$ in terms of $k^{d}$, the dispersion error using flexible integration point in the MR-FEM can be written as follows:

$$
k=k^{d}-\frac{\left(k^{d}\right)^{3} d^{2}}{24}\left(2 \cos ^{4} \varphi-2 \cos ^{2} \varphi+\left(3 q^{2}-2 q-1\right) \sin 2 \varphi+6 q^{2}-4 q-1\right)+o\left(\left(k^{d}\right)^{5}\right)
$$

The relative dispersion error $e(\varphi, k)$ is defined as the difference between the actual wavenumber and the approximate one: 


$$
e(\varphi, k)=\left|\frac{k^{e x}-k^{d}}{k^{d}}\right|
$$

where $k^{d}$ is the approximate wavenumber, and $k^{e x}$ represents the actual wavenumber.

The dispersion error of MR-FEM can be quantified as the relative error which is described as follows:

$$
e(\varphi, k)=\left|\frac{\left(k^{d}\right)^{2} d^{2}}{24}\left(2 \cos ^{4} \varphi-2 \cos ^{2} \varphi+\left(3 q^{2}-2 q-1\right) \sin 2 \varphi+6 q^{2}-4 q-1\right)+o\left(\left(k^{d}\right)^{4}\right)\right|
$$

As the dispersion error is small, $k \approx k^{d}$, and

$$
2\left(\cos ^{4} \varphi-\cos ^{2} \varphi\right)=2 \cos ^{2} \varphi\left(\cos ^{2} \varphi-1\right)=-2 \cos ^{2} \varphi \sin ^{2} \varphi=-\frac{1}{2} \sin ^{2} 2 \varphi
$$

The relative dispersion error is finally expressed as follows:

$$
e(\varphi, k)=\left|\frac{k^{2} d^{2}}{24}\left(-\frac{1}{2} \sin ^{2} 2 \varphi+\left(3 q^{2}-2 q-1\right) \sin 2 \varphi+6 q^{2}-4 q-1\right)+o\left(k^{4}\right)\right|
$$

It is apparently noticed that the dispersion error is minimized as the term of $6 q^{2}-4 q-1$ in Eq. (49) is equal to zero.

$$
e(\varphi, k)=\left|\frac{k^{2} d^{2}}{24}\left(-\frac{1}{2} \sin ^{2} 2 \varphi-\frac{1}{2} \sin 2 \varphi\right)+o\left(k^{4}\right)\right|
$$

Thus, the optimal value of $q$ is: $q=\frac{4 \pm \sqrt{16+4 \times 6}}{12}=\frac{2 \pm \sqrt{10}}{6}$.

Based on the optimal integration points, it is expected that the discretized system for fluid domain is optimally balanced. Thus, the dispersion error is minimized compared with traditional Gauss integration points. The outstanding features of MR-FEM via several numerical examples are presented in Section 4.

\section{Numerical examples}


In this section, the wave propagation in the fluid/solid and solid/fluid PCs with FSI using MR-FEM and standard FEM is investigated. In this part, the accuracy and computational efficiency of MR-FEM are studied in detail. It is expected that this novel formulation of MR-FEM could be an effective tool to analyze and design PCs with multifunction properties.

To investigate quantitatively the numerical results, the error indicator Error of eigenfrequency in wave vector is defined as follows:

$$
\text { Error }=\sqrt{\frac{\sum_{i=1}^{n}\left(\xi_{i}^{\text {num }}-\xi_{i}^{\text {exact }}\right)^{2}}{n \sum_{i=1}^{n}\left(\xi_{i}^{\text {exact }}\right)^{2}}}
$$

where $\xi^{\text {num }}$ represents the numerical solution of eigenfrequency obtained from MR-

FEM and standard FEM, $n$ is the number of eigenfrequency, and $\xi^{\text {exact }}$ stands for the exact solution of eigenfrequency or the reference solution obtained from COMSOL using very fine mesh. In all the numerical examples, the materials for the solid and fluid are aluminum and water, respectively. The material properties for aluminum and water are taken as follows:

$$
\begin{gathered}
\rho_{s}=2700 \mathrm{~kg} / \mathrm{m}^{3}, E=71 \mathrm{Gpa}, v=0.3 \\
\rho_{f}=1000 \mathrm{~kg} / \mathrm{m}^{3}, c=1500 \mathrm{~m} / \mathrm{s}
\end{gathered}
$$

\subsection{Solid/fluid PCs with square lattice}

As shown in Fig. 5, a square lattice with circular scatterers is considered. The lattice constant $a=0.15 \mathrm{~m}$, and the radius for the circular scatterers is $r=0.05 \mathrm{~m}$. Fig. 6 compares the band gap computed by standard FEM and MR-FEM using the same number of nodes (solid: 23 nodes, 31 triangular elements; fluid: 55 nodes, 73 triangular elements). The 
reference solutions are obtained using commercial software COMSOL with fine mesh (solid: 1483 nodes; fluid: 1862 nodes). As outlined in Fig. 6, the band gap obtained from MR-FEM is almost identical to the reference solution. In contrast to MR-FEM model, there is a large difference of band gap between standard FEM and the reference models.

The study of convergence rate for first fifteen eigenfrequencies (along $\mathrm{X}, \mathrm{M}$ and $\Gamma$ directions) using MR-FEM and FEM models is conducted in Fig. 7. Four types of mesh are used in both MR-FEM and FEM models using triangular elements as shown in Table 1. The reference solutions are obtained using commercial software COMSOL (1483 nodes for solid, 1862 nodes for fluid). It is clearly seen that all numerical errors from MR-FEM and FEM models reduce with increasing number of nodes in the discretized domain. However, the MR-FEM always gives much better accuracy compared with standard FEM using the same mesh.

The computational time is always a critical issue in the development of efficient numerical methods. As depicted in Fig. 8, it is clearly seen that the computational time for MR-FEM and FEM is almost the same for different types of set of mesh. However, in comparison of computational efficiency, it is found that MR-FEM shows very excellent advantages compared with standard FEM as outlined in Fig.9. This is very important to simulate large scale problems using MR-FEM.

\subsection{Helmholtz resonator PCs with square lattice}

The second example is Helmholtz resonator PCs with square lattice as shown in Fig. 10. The lattice constant $a=0.1 \mathrm{~m}$ is taken in this example. The band gap obtained from FEM and MR-FEM using coarse mesh (30 nodes for solid, 82 nodes for fluid) is presented in Fig. 11. The reference solution is obtained using commercial software using 
COMSOL (637 nodes for solid, 2424 nodes for fluid). As expected, the prediction of band gap from MR-FEM has an excellent agreement with that using commercial software, which has strongly validated the accuracy of MR-FEM again. This is due to an optimal balance in the stiffness and mass with tuning parameter $q$ of integration point. On the contrary, there is a large deviation of numerical results between standard FEM and reference models.

Figure 12 studies the convergence rate of standard FEM and MR-FEM for the computation of the first fifteen eigenfrequencies along $\mathrm{X}, \mathrm{M}$ and $\Gamma$ directions. Three sets of mesh as outlined in Table 2 are employed in the convergence analysis. Again, it is noticed that all the numerical solutions in both FEM and MR-FEM models converge to the reference solution as the average distance between nodes decreases. However, the accuracy of MR-FEM is always more accurate compared with standard FEM with the same number of nodes.

The computational efficiency of standard FEM and MR-FEM models for first fifteen eigenfrequencies is illustrated in Fig. 13. It is noticed that the MR-FEM stands out clearly compared with standard FEM. First of all, the computational cost for both standard FEM and MR-FEM is almost identical due to the similar bandwidth. However, the MR-FEM always gives much better accuracy, as depicted in Fig. 13. The excellent feature of MRFEM has been demonstrated again by this example.

\subsection{Solid/fluid PCs with triangular lattice}

Next, we discuss the solid/fluid PCs with triangular lattice as outlined in Fig. 14. The lattice constant $a=0.086603 \mathrm{~m}$, and the aluminum scatterers $b=0.05 \mathrm{~m}$. Fig. 15 shows the numerical results of band gap computed by standard FEM and MR-FEM using coarse 
mesh (solid: 64 nodes, 104 triangular elements; fluid: 62 nodes, 72 triangular elements). The reference solutions are obtained using commercial software COMSOL (solid: 1169 nodes, 2204 triangular elements; fluid: 2081 nodes, 3832 triangular elements). As expected, the numerical solutions from MR-FEM always have a fair agreement with the reference solutions. However, the standard FEM solutions become worse at the high frequency mode due to the overly-stiff property.

The convergence rate of MR-FEM and standard FEM for the first fifteen eigenfrequencies is shown in Fig. 16. Four types of set of mesh as shown in Table 3 are used in the study. It is obviously seen that MR-FEM always gives much better solutions compared with standard FEM using the same type of mesh. This example has clearly validated the outstanding advantages of MR-FEM in the simulation of fluid/solid PCs.

\subsection{Fluid/Solid PCs with square lattice}

This subsection discusses the computational efficiency of MR-FEM in the analysis of fluid/solid PCs with square lattice as outlined in Fig. 17. In this example, the square lattice constant $a=0.15 \mathrm{~m}$, and the size of square water scatterers $b=0.1 \mathrm{~m}$. The numerical solutions of band gap using MR-FEM and standard FEM with the same type of mesh (solid: 104 nodes, 140 triangular elements; fluid: 71 nodes, 112 triangular elements) are plotted in Fig. 18. For the purpose of comparison, the reference solutions are computed from COMSOL using very fine mesh (solid: 1508 nodes, 1923 triangular elements; fluid: 1023 nodes, 1428 triangular elements). As outlined in Fig. 18, compared with overly-stiff FEM model, the MR-FEM solutions are very close to the reference solutions, especially at high frequency mode shape. 
The comparison of convergence rate for the first fifteen eigenfrequencies (along $\mathrm{X}$, $M$ and $\Gamma$ directions) using MR-FEM and standard FEM models is depicted in Fig. 19. Three sets of mesh as outlined in Table 4 are used in this study. Again, MR-FEM produces much better accuracy compared with standard FEM. The optimal balance between the stiffness and mass in the MR-FEM has been validated again, which shows very promising feature in the simulation of fluid/solid PCs with square lattice.

\subsection{Fluid/Solid PCs with triangular lattice}

Figure 20 shows the fluid/solid PCs with triangular lattice, and the water scatterers are embedded in the aluminum matrix. In the current analysis, the lattice constant $a=0.086603 \mathrm{~m}$, and the size of square water scatterers $b=0.05 \mathrm{~m}$. Fig. 21 shows the numerical results of band gap of fluid/solid PCs with triangular lattice. It is noted that the standard FEM and MR-FEM models use the same coarse mesh (solid: 96 nodes, 118 triangular elements; fluid: 40 nodes, 58 triangular elements), and the reference solutions are obtained using very fine mesh (solid: 2081 nodes, 3832 triangular elements; fluid: 1169 nodes, 2204 triangular elements). It is obviously noticed that the numerical solutions from MR-FEM have a fair agreement with the reference solutions from lower to higher mode orders. However, a large deviation between the FEM and the reference models is clearly observed in Fig. 21.

The comparison of convergence rate in both standard FEM and MR-FEM models for the first fifteen eigenfrequencies (along X, M and $\Gamma$ directions) is studied in Fig. 22. Four different types of mesh as shown in Table 5 are employed in this study. Obviously, the accuracy MR-FEM is much better than the standard FEM. 


\subsection{Extreme case with mercury/rubber}

The last example presents an extreme case: rubber is immersed into mercury as shown in Fig. 23. The radius for the circular scatterers is $r=0.05 \mathrm{~m}$, and the lattice constant $a=0.15 \mathrm{~m}$. It is well known the density of mercury is very high compared with water, and rubber is considered a relatively soft material. The properties of mercury and rubber are taken as follows:

$$
\begin{gathered}
\rho_{r u b}=1000 \mathrm{~kg} / \mathrm{m}^{3}, E=730 \mathrm{Mpa}, v=0.4597 \\
\rho_{\text {Mer }}=13600 \mathrm{~kg} / \mathrm{m}^{3}, c=1451 \mathrm{~m} / \mathrm{s}
\end{gathered}
$$

Figure 24 presents the band gap using FEM and MR-FEM with the same mesh (123 nodes and 212 triangular elements for rubber; 194 nodes and 296 triangular elements for mercury). The reference solutions using very fine mesh (1645 nodes for rubber; 3016 nodes for mercury) are also plotted for the purpose of compassion. Again, it is found that numerical results obtained from MR-FEM are still better that those from standard FEM. This example has clearly validated the robustness of MR-FEM.

\section{Discussions and Conclusions}

In this work, with employment of flexible integration points, a general mass matrix is formulated to study the wave propagation in the phononic crystals (PCs) with fluid structure interaction (FSI). Instead of using traditional Gauss integration point, the optimal integration point in the formulation of mass matrix based on the dispersion error analysis has created an optimal balance between the stiffness and mass in the fluid domain. Such tuning can be easily done without increasing computational cost and additional computational procedure. A number of numerical examples including fluid/solid and solid/fluid PCs with square and triangular lattices have clearly 
demonstrated the accuracy and computational efficiency of the proposed MR-FEM to analyze the wave propagation of in the 2D PCs with FSI. The novel, accurate, robust and efficient MR-FEM with combination of advanced topology optimization has the great potential to provide an effective tool to design the 3D multifunctional fluid/solid and solid/fluid PCs.

\section{Acknowledgements}

The authors also wish to thank Research Project of the Science Fund of State Key Laboratory of Advanced Design and Manufacturing for Vehicle Body (Grant No. 31615002), National Natural Science Foundation of Hunan (Grant No. 2017JJ3030), and Research Project of State Key Laboratory of Structural Analysis for Industrial Equipment (Grant No. GZ 1609) for the support. 


\section{Reference}

[1] Tang Y, Yin J. Design of cut unit geometry in hierarchical kirigami-based auxetic metamaterials for high stretchability and compressibility. Extreme Mechanics Letters. 2016.

[2] Yuan JH, Lu YY, Antoine X. Modeling photonic crystals by boundary integral equations and Dirichlet-to-Neumann maps. Journal of Computational Physics. 2008;227:4617-29.

[3] Gatt R, Mizzi L, Azzopardi JI, Azzopardi KM, Attard D, Casha A, et al. Hierarchical Auxetic Mechanical Metamaterials. Scientific Reports. 2015;5.

[4] Grima JN, Mizzi L, Azzopardi KM, Gatt R. Auxetic Perforated Mechanical Metamaterials with Randomly Oriented Cuts. Advanced Materials. 2016;28:385-9.

[5] Florijn B, Coulais C, van Hecke M. Programmable mechanical metamaterials: the role of geometry. Soft Matter. 2016;12:8736-43.

[6] Zhou SW, Li W, Chen YH, Sun GY, Li Q. Topology optimization for negative permeability metamaterials using level-set algorithm. Acta Materialia. 2011;59:2624-36.

[7] Liu YM, Zhang X. Metamaterials: a new frontier of science and technology. Chemical Society Reviews. 2011;40:2494-507.

[8] Liu AQ, Zhu WM, Tsai DP, Zheludev NI. Micromachined tunable metamaterials: a review. Journal of Optics. 2012;14.

[9] Brunet T, Leng J, Mondain-Monval O. Soft Acoustic Metamaterials. Science. 2013;342:323-4. [10] Viktor GV. The electrodynamics of substances with simultaneously negative values of $\epsilon$ and $\mu$. Soviet Physics Uspekhi. 1968;10:509.

[11] Liu XN, Hu GK, Huang GL, Sun CT. An elastic metamaterial with simultaneously negative mass density and bulk modulus. Applied Physics Letters. 2011;98.

[12] Shelby RA, Smith DR, Schultz S. Experimental verification of a negative index of refraction. Science. 2001;292:77-9.

[13] Huang GL, Sun CT. Band Gaps in a Multiresonator Acoustic Metamaterial. Journal of Vibration and Acoustics-Transactions of the Asme. 2010;132.

[14] Huang HH, Sun CT, Huang GL. On the negative effective mass density in acoustic metamaterials. International Journal of Engineering Science. 2009;47:610-7.

[15] Tan KT, Huang HH, Sun CT. Optimizing the band gap of effective mass negativity in acoustic metamaterials. Applied Physics Letters. 2012;101.

[16] Sigalas MM, Economou EN. Elastic and Acoustic-Wave Band-Structure. Journal of Sound and Vibration. 1992;158:377-82.

[17] Zhu R, Huang HH, Huang GL, Sun CT. Microstructure continuum modeling of an elastic metamaterial. International Journal of Engineering Science. 2011;49:1477-85. 
[18] Chen HJ, Li H, Zhai SL, Ding CL, Li JM, Luo CR, et al. Ultrasound acoustic metamaterials with double-negative parameters. Journal of Applied Physics. 2016;119.

[19] Krushynska AO, Kouznetsova VG, Geers MGD. Visco-elastic effects on wave dispersion in three-phase acoustic metamaterials. Journal of the Mechanics and Physics of Solids. 2016;96:2947.

[20] Song GY, Cheng Q, Huang B, Dong HY, Cui TJ. Broadband fractal acoustic metamaterials for low-frequency sound attenuation. Applied Physics Letters. 2016;109.

[21] Zhou XQ, Wang J, Wang RQ, Lin JQ. Effects of relevant parameters on the bandgaps of acoustic metamaterials with multi-resonators. Applied Physics a-Materials Science \& Processing. $2016 ; 122$.

[22] He ZC, Xiao X, Li E. Design for structural vibration suppression in laminate acoustic metamaterials. Composites Part B: Engineering. 2017;131:237-52.

[23] Li E, He ZC, Hu JY, Long XY. Volumetric locking issue with uncertainty in the design of locally resonant acoustic metamaterials. Computer Methods in Applied Mechanics and Engineering. 2017;324:128-48.

[24] Huang HH, Sun CT. Wave attenuation mechanism in an acoustic metamaterial with negative effective mass density. New Journal of Physics. 2009;11.

[25] Zhu R, Huang GL, Hu GK. Effective Dynamic Properties and Multi-Resonant Design of Acoustic Metamaterials. Journal of Vibration and Acoustics-Transactions of the Asme. 2012;134.

[26] Liu ZY, Zhang XX, Mao YW, Zhu YY, Yang ZY, Chan CT, et al. Locally resonant sonic materials. Science. 2000;289:1734-6.

[27] Zhu R, Liu XN, Hu GK, Sun CT, Huang GL. A chiral elastic metamaterial beam for broadband vibration suppression. Journal of Sound and Vibration. 2014;333:2759-73.

[28] Li J, Chan CT. Double-negative acoustic metamaterial. Physical Review E. 2004;70.

[29] Liu Y, Su X, Sun CT. Broadband elastic metamaterial with single negativity by mimicking lattice systems. Journal of the Mechanics and Physics of Solids. 2015;74:158-74.

[30] Chen YY, Huang GL, Sun CT. Band Gap Control in an Active Elastic Metamaterial With Negative Capacitance Piezoelectric Shunting. Journal of Vibration and Acoustics-Transactions of the Asme. 2014;136.

[31] Li. YF, Huang. X, Zhou. S. Topological Design of Cellular Phononic Band Gap Crystals. Materials. 2016;9:186.

[32] Wu J, Luo Z, Li H, Zhang N. Level-set topology optimization for mechanical metamaterials under hybrid uncertainties. Computer Methods in Applied Mechanics and Engineering. 2017;319:414-41. 
[33] Meng F, Li S, Lin H, Jia B, Huang X. Topology optimization of photonic structures for allangle negative refraction. Finite Elements in Analysis and Design. 2016;117:46-56.

[34] Li FL, Wang YS, Zhang CZ. Bandgap calculation of two-dimensional mixed solid-fluid phononic crystals by Dirichlet-to-Neumann maps. Physica Scripta. 2011;84.

[35] He ZC, Li E, Wang G, Li GY, Xia Z. Development of an efficient algorithm to analyze the elastic wave in acoustic metamaterials. Acta Mechanica. 2016;227:3015-30.

[36] Li E, He ZC, Wang G. An exact solution to compute the band gap in phononic crystals. Computational Materials Science. 2016;122:72-85.

[37] Li E, He ZC, Wang G, Liu GR. An ultra-accurate numerical method in the design of liquid phononic crystals with hard inclusion. Computational Mechanics. 2017.

[38] Zheng H, Zhang C, Wang Y, Sladek J, Sladek V. Band structure computation of in-plane elastic waves in 2D phononic crystals by a meshfree local RBF collocation method. Engineering Analysis with Boundary Elements. 2016;66:77-90.

[39] Vogiatzis P, Ma M, Chen S, Gu XD. Computational design and additive manufacturing of periodic conformal metasurfaces by synthesizing topology optimization with conformal mapping. Computer Methods in Applied Mechanics and Engineering. 2018;328:477-97.

[40] Xiao SS, Shen LF, He SL. A plane-wave expansion method based on the effective medium theory for calculating the band structure of a two-dimensional photonic crystal. Physics Letters A. 2003;313:132-8.

[41] Shi SY, Chen CH, Prather DW. Plane-wave expansion method for calculating band structure of photonic crystal slabs with perfectly matched layers. Journal of the Optical Society of America a-Optics Image Science and Vision. 2004;21:1769-75.

[42] Zhao Y, Belov P, Hao Y. Accurate modelling of left-handed metamaterials using a finitedifference time-domain method with spatial averaging at the boundaries. Journal of Optics a-Pure and Applied Optics. 2007;9:S468-S75.

[43] Zheng H, Zhang CZ, Wang YS, Sladek J, Sladek V. A meshfree local RBF collocation method for anti-plane transverse elastic wave propagation analysis in $2 \mathrm{D}$ phononic crystals. Journal of Computational Physics. 2016;305:997-1014.

[44] Yan ZZ, Wang YS. Wavelet-based method for calculating elastic band gaps of twodimensional phononic crystals. Physical Review B. 2006;74.

[45] Li JB, Wang YS, Zhang CZ. Dispersion Relations Of a Periodic Array Of Fluid-Filled Holes Embedded In an Elastic Solid. Journal of Computational Acoustics. 2012;20.

[46] Li JB, Wang YS, Zhang CZ. Tuning of Acoustic Bandgaps in Phononic Crystals With Helmholtz Resonators. Journal of Vibration and Acoustics-Transactions of the Asme. 2013;135. 
[47] Liu Y, Gao LT. Explicit dynamic finite element method for band-structure calculations of 2D phononic crystals. Solid State Communications. 2007;144:89-93.

[48] Li FL, Wang YS, Zhang CZ, Yu GL. Bandgap calculations of two-dimensional solid-fluid phononic crystals with the boundary element method. Wave Motion. 2013;50:525-41.

[49] Shi ZJ, Wang YS, Zhang CZ. Application of the generalized multipole technique in band structure calculation of two-dimensional solid/fluid phononic crystals. Mathematical Methods in the Applied Sciences. 2015;38:3246-63.

[50] Shi ZJ, Wang YS, Zhang CZ. Band structure calculations of in-plane waves in twodimensional phononic crystals based on generalized multipole technique. Applied Mathematics and Mechanics-English Edition. 2015;36:557-80.

[51] Zheng H, Zhang CZ, Wang YS, Chen W, Sladek J, Sladek V. A local RBF collocation method for band structure computations of $2 \mathrm{D}$ solid/fluid and fluid/solid phononic crystals. International Journal for Numerical Methods in Engineering. 2017;110:467-500.

[52] He ZC, Li E, Liu GR, Li GY, Cheng AG. A mass-redistributed finite element method (MRFEM) for acoustic problems using triangular mesh. Journal of Computational Physics. 2016;323:149-70.

[53] Li E, He ZC, Jiang Y, Li B. 3D mass-redistributed finite element method in structuralacoustic interaction problems. Acta Mechanica. 2016;227:857-79.

[54] Liu GR, Dai KY, Nguyen TT. A smoothed finite element method for mechanics problems. Computational Mechanics. 2007;39:859-77.

[55] Liu GR, Nguyen TT. Smoothed finite element methods. Boca Raton: Taylor \& Francis,; 2010. p. 691 p.

[56] Li E, He ZC, Xu X, Liu GR. Hybrid smoothed finite element method for acoustic problems. Computer Methods in Applied Mechanics and Engineering. 2015;283:664-88.

[57] Li E, Zhang ZP, Chang CC, Liu GR, Li Q. Numerical homogenization for incompressible materials using selective smoothed finite element method. Composite Structures. 2015;123:21632.

[58] He Z, Li G, Zhang G, Liu G-R, Gu Y, Li E. Acoustic analysis using a mass-redistributed smoothed finite element method with quadrilateral mesh. Engineering Computations. 2015;32:2292-317.

[59] Guddati MN, Yue B. Modified integration rules for reducing dispersion error in finite element methods. Computer Methods in Applied Mechanics and Engineering. 2004;193:275-87.

[60] Yue B, Guddati MN. Dispersion-reducing finite elements for transient acoustics. Journal of the Acoustical Society of America. 2005;118:2132-41. 
[61] Li E, He ZC, Xu X, Zhang GY, Jiang Y. A faster and accurate explicit algorithm for quasiharmonic dynamic problems. International Journal for Numerical Methods in Engineering. 2016;108:839-64.

[62] Li E, He ZC, Zhang Z, Liu GR, Li Q. Stability analysis of generalized mass formulation in dynamic heat transfer. Numerical Heat Transfer, Part B: Fundamentals. 2016;69:287-311.

[63] Wang G, Wen JH, Liu YZ, Wen XS. Lumped-mass method for the study of band structure in two-dimensional phononic crystals. Physical Review B. 2004;69.

[64] Li E, Liao WH. An Efficient Finite Element Algorithm in Elastography. International Journal of Applied Mechanics. 2016;8.

[65] Ashcroft NW, Mermin ND. Solid state physics. New York: , Holt, 1976. 
Figure

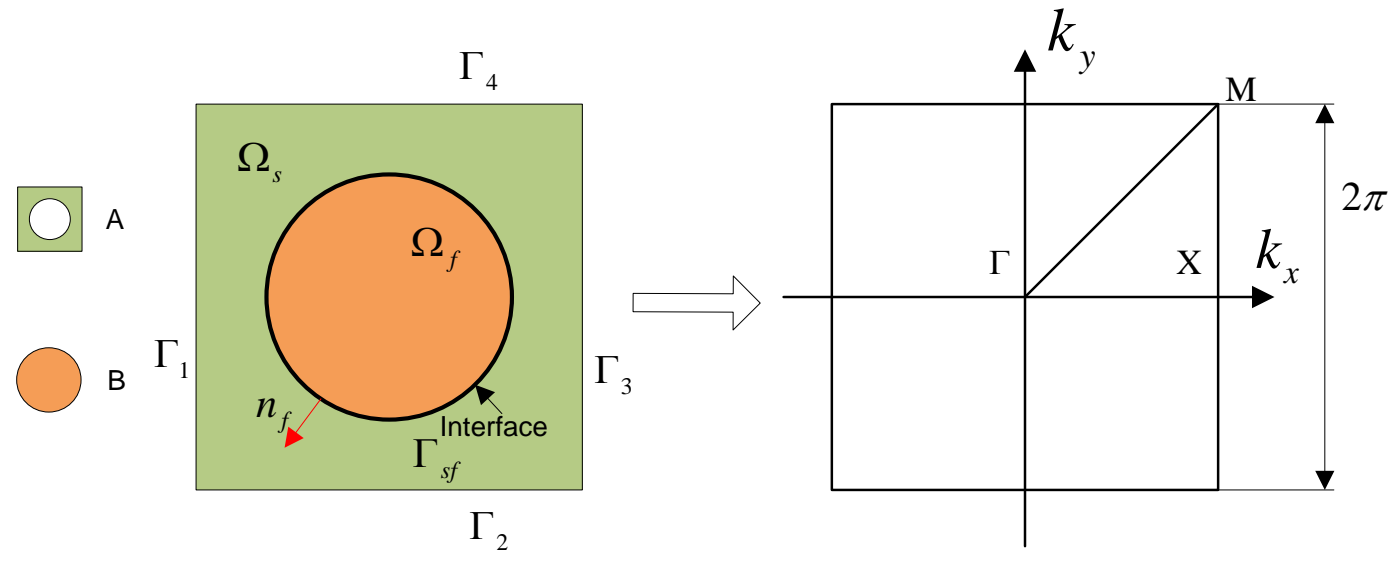

a) Periodic square cell

b) First Brillouinzone

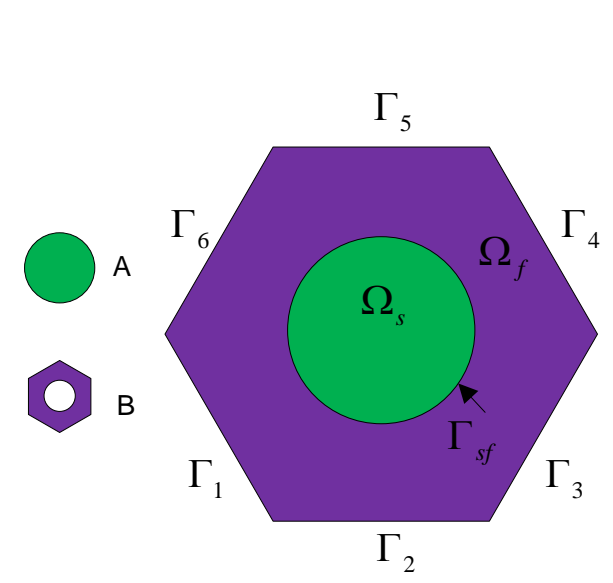

c) Periodic hexagonal unit cell

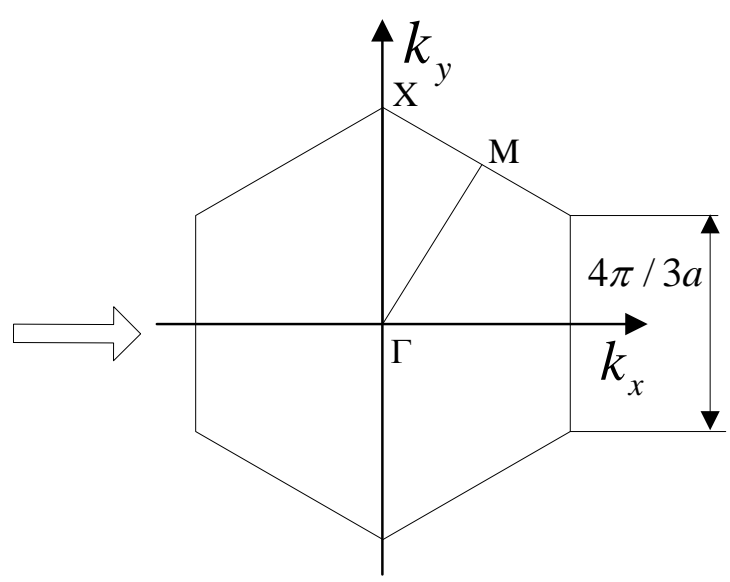

d) First Brillouinzone

Figure 1: Fluid/solid and solid/fluid PCs 


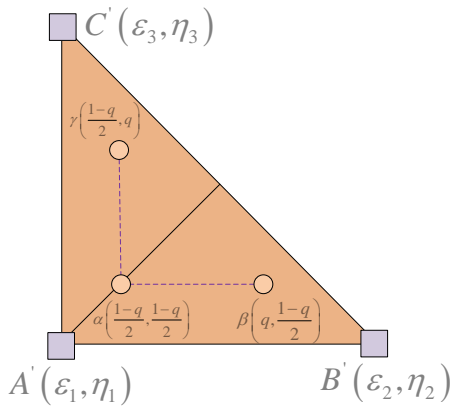

a) MR-FEM

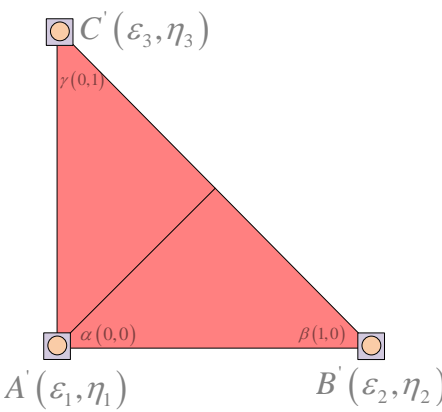

b) $q=1$ Lumped mass matrix

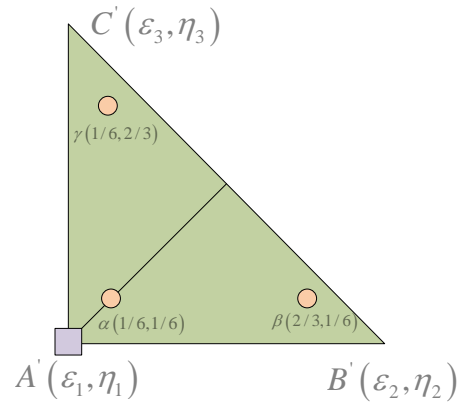

c) $q=2 / 3$ Consistent mass matrix

Integration point $\square \quad$ Nodes of field

Figure 2: Formulation of MR-FEM 


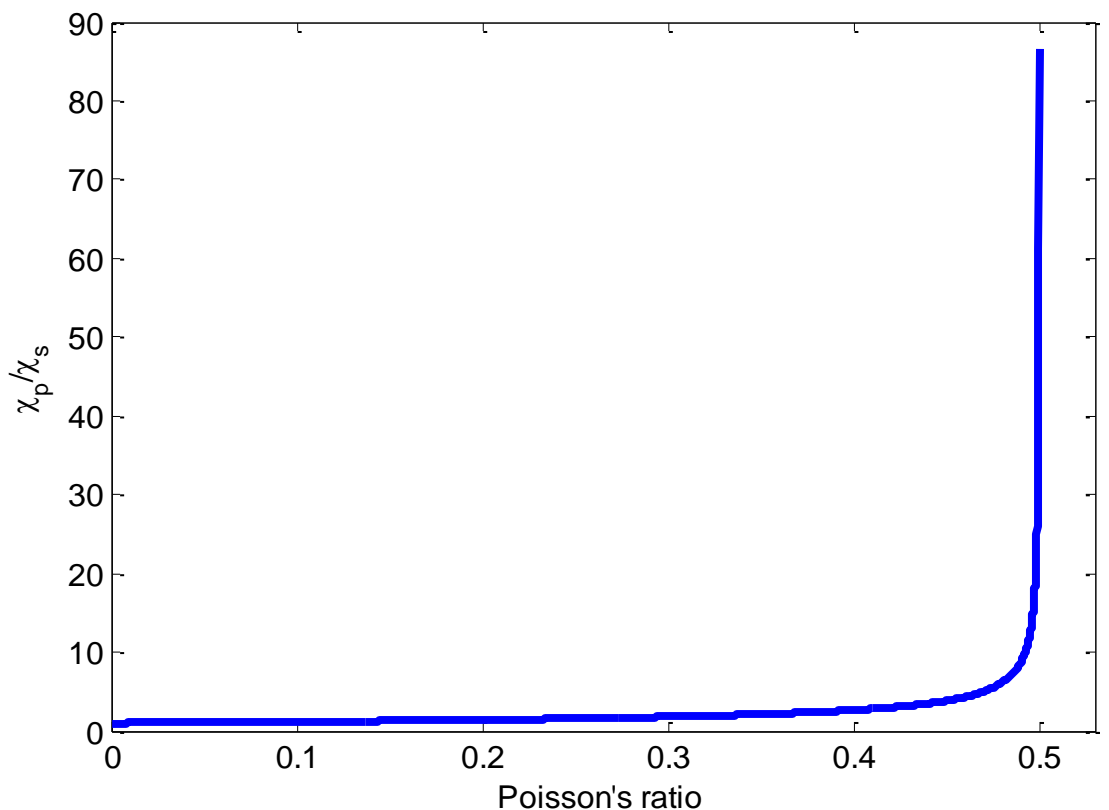

Figure 3: The ratio between the pressure and shear wave versus Poisson's ratio in the solid domain 


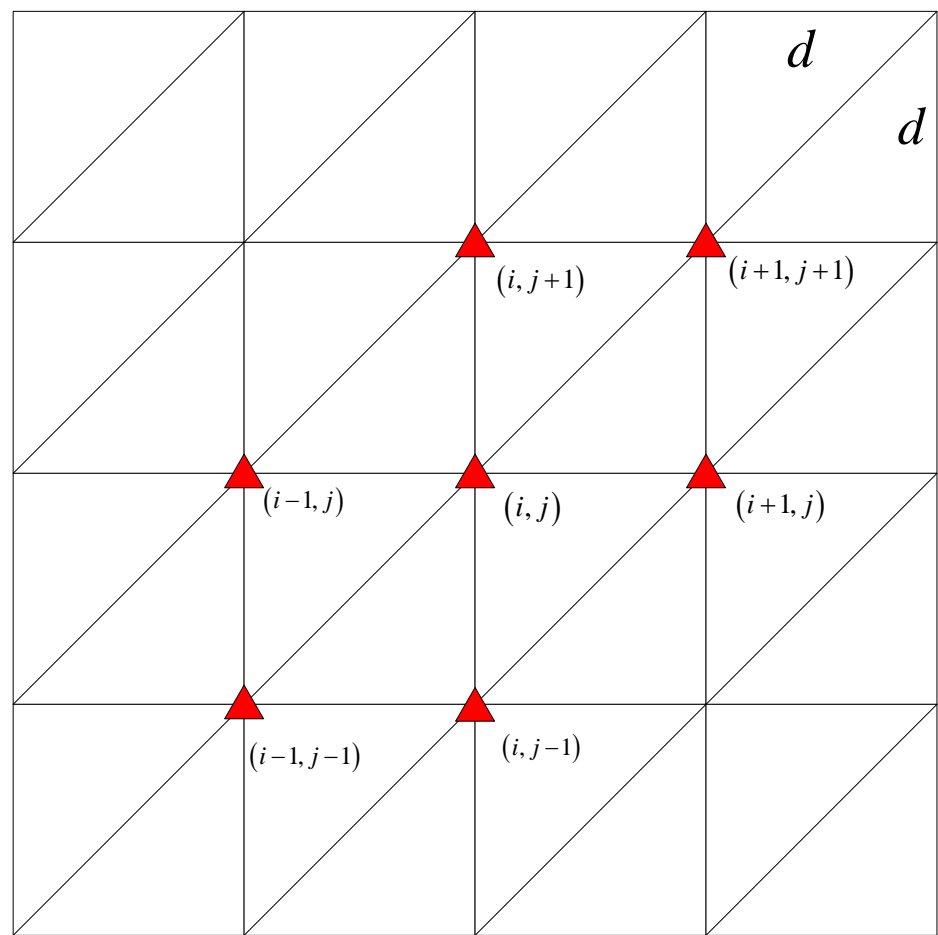

Figure 4: Dispersion error analysis in the framework of MR-FEM 


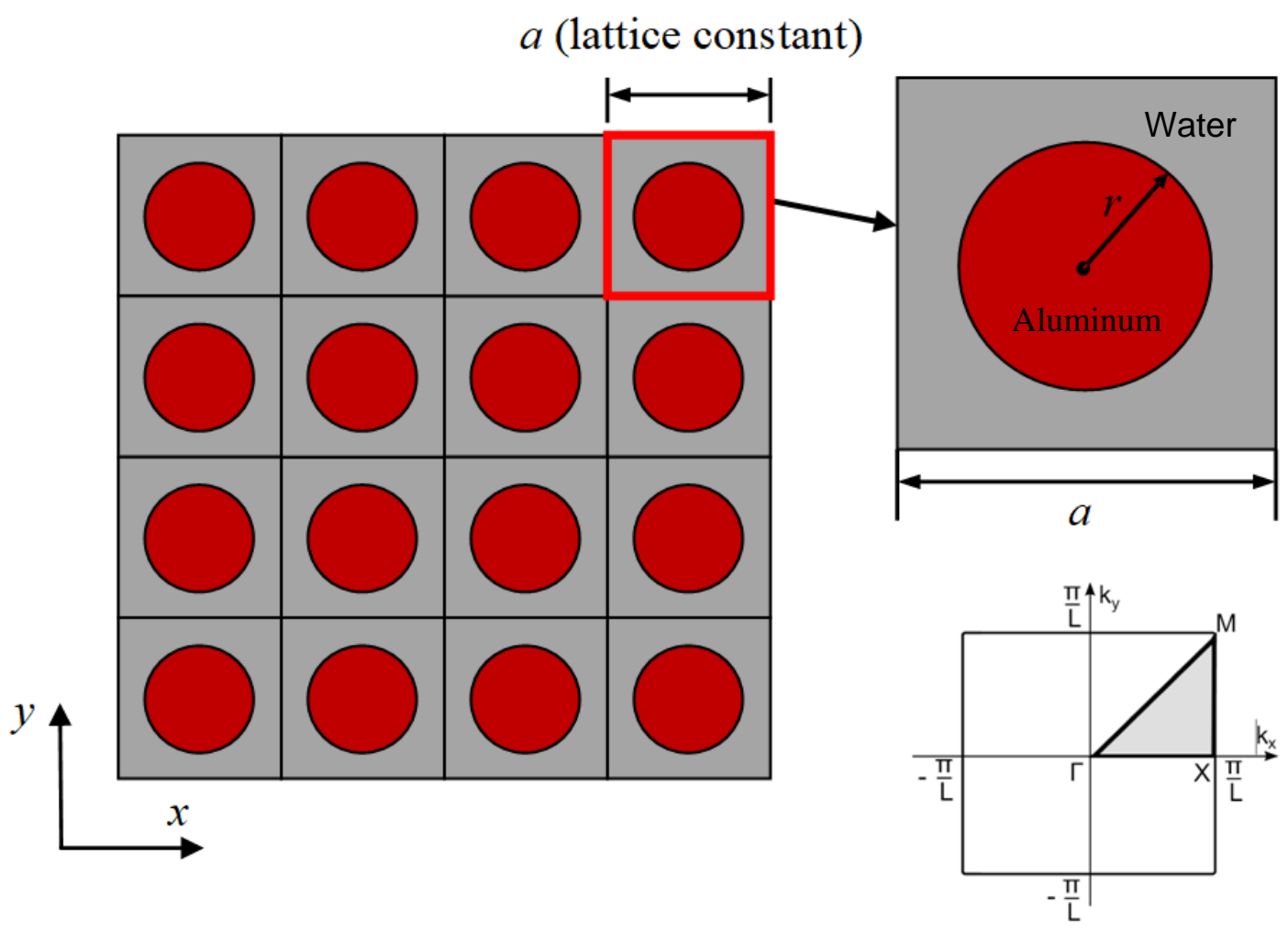

Figure 5: Solid/fluid PCs with square lattice 


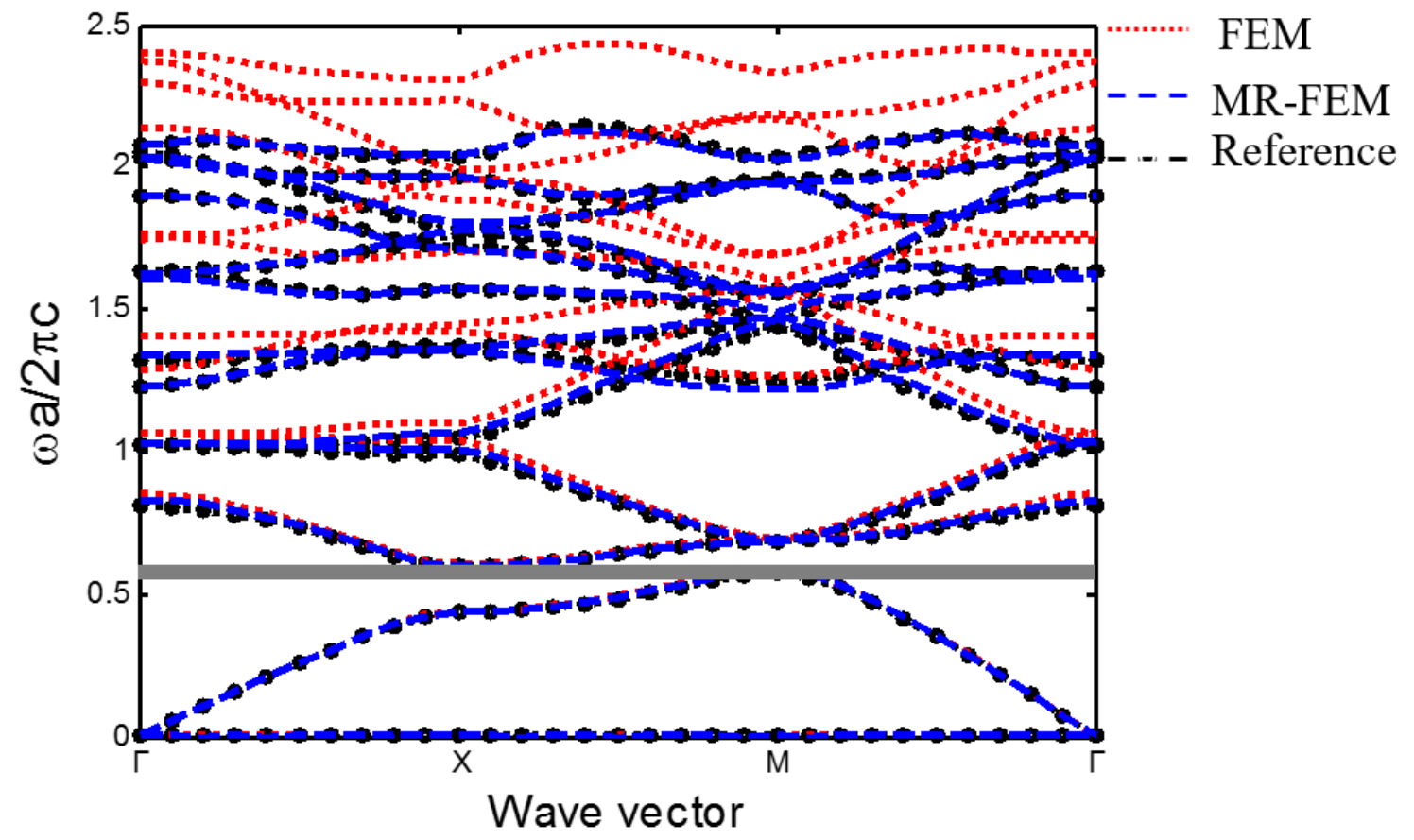

Figure 6: Comparison of band gap using different numerical methods 


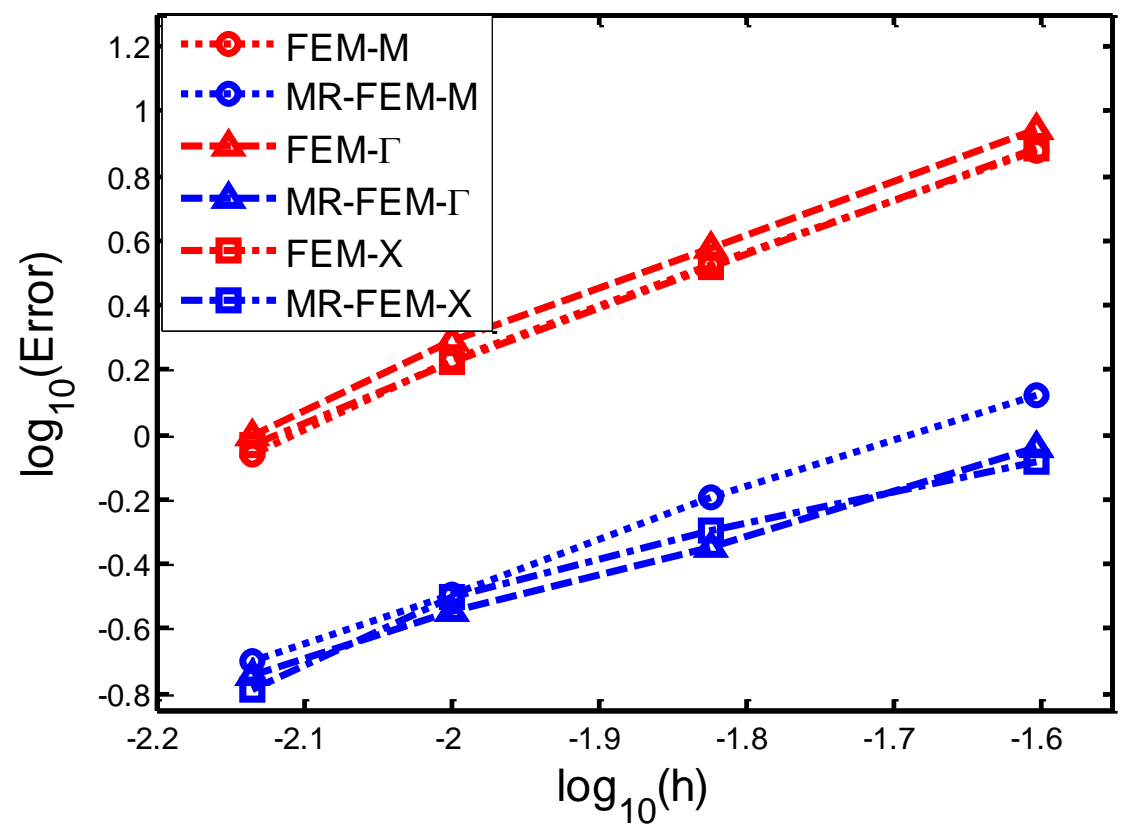

Figure 7: Comparison of convergence rate 


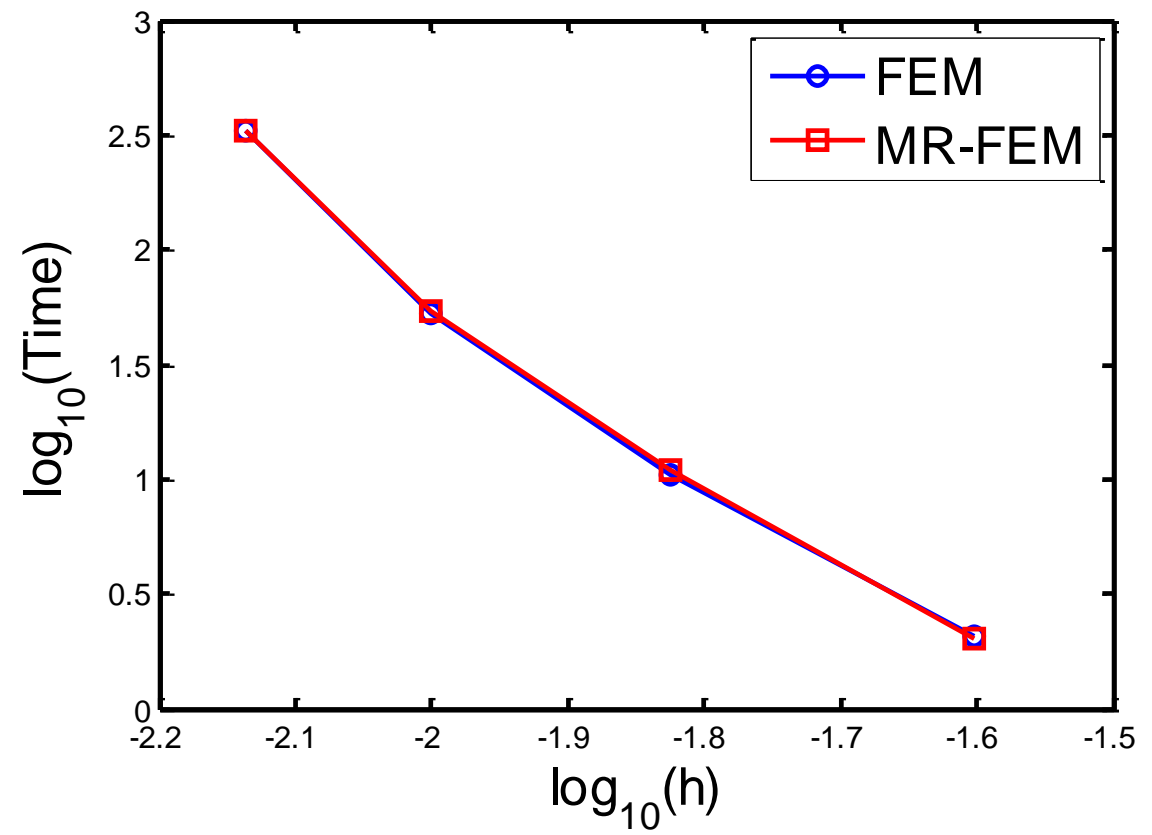

Figure 8: Comparison of computational time 


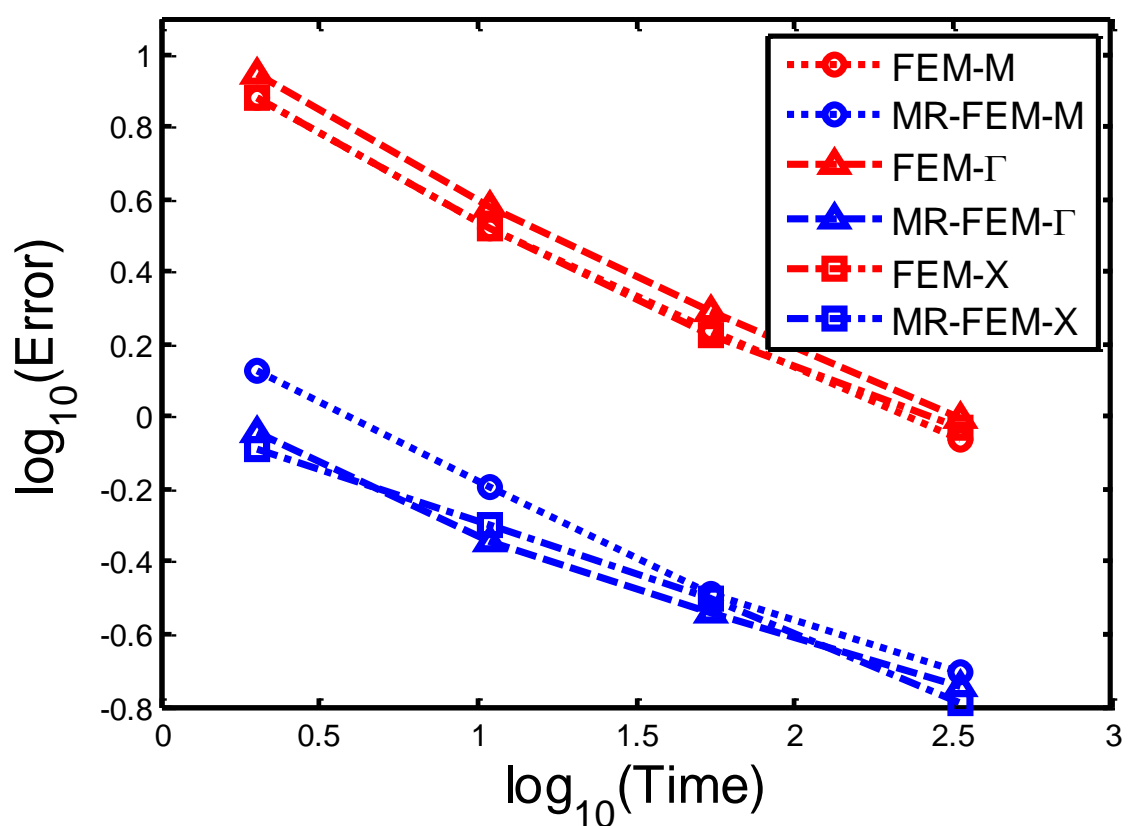

Figure 9: Computational efficiency 


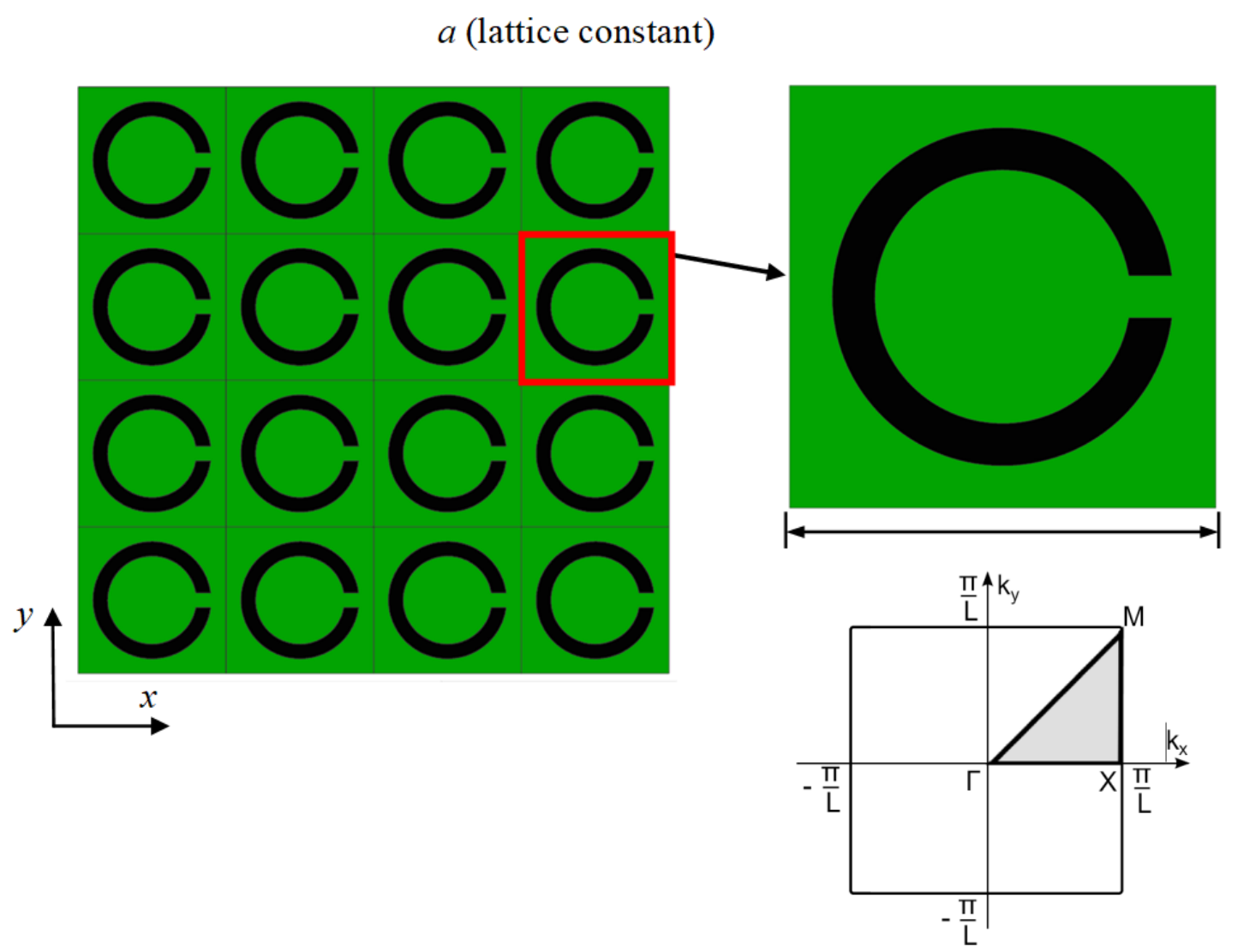

Figure 10: Helmholtz resonator PCs with square lattice 


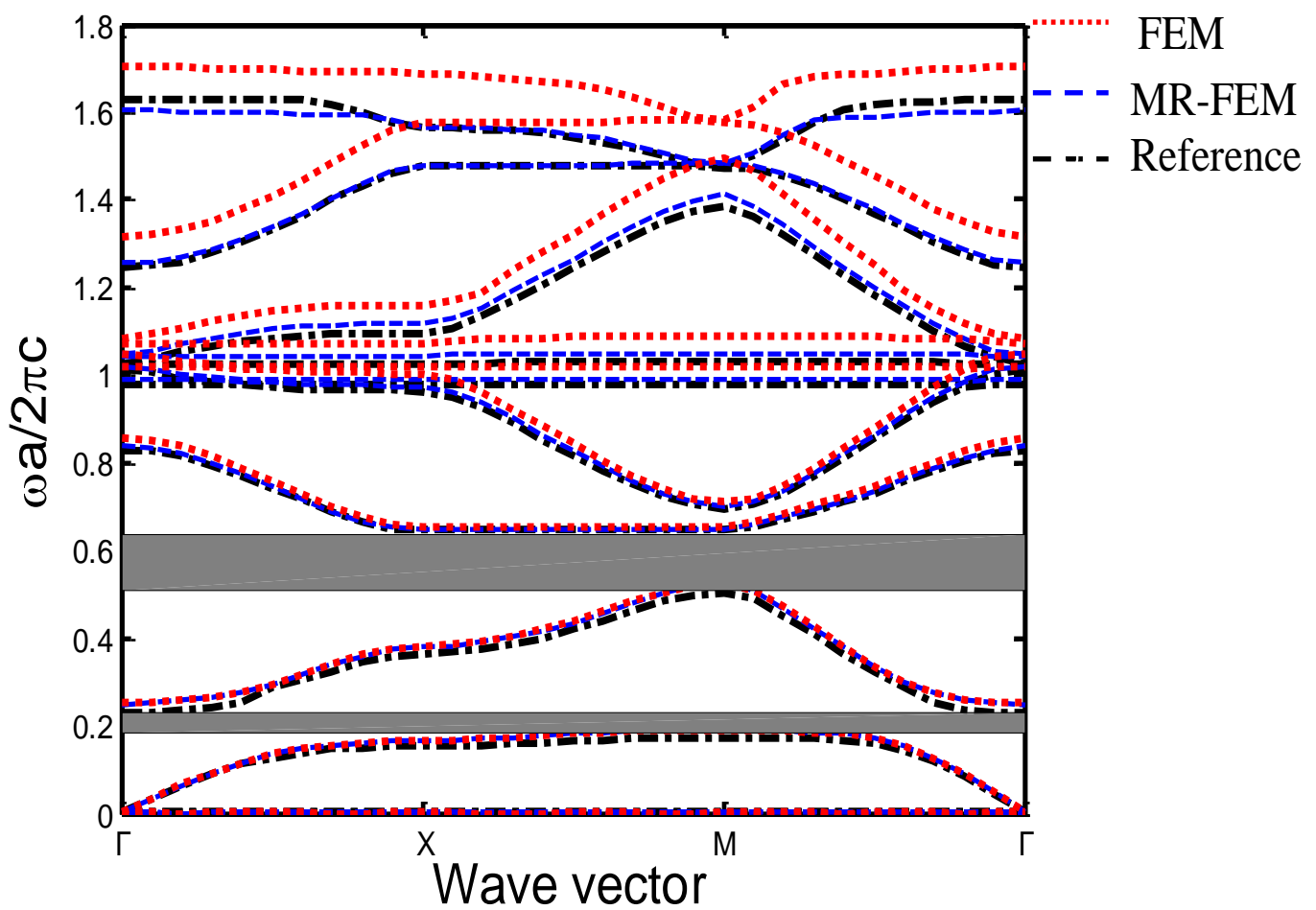

Figure 11: Comparison of band gap 


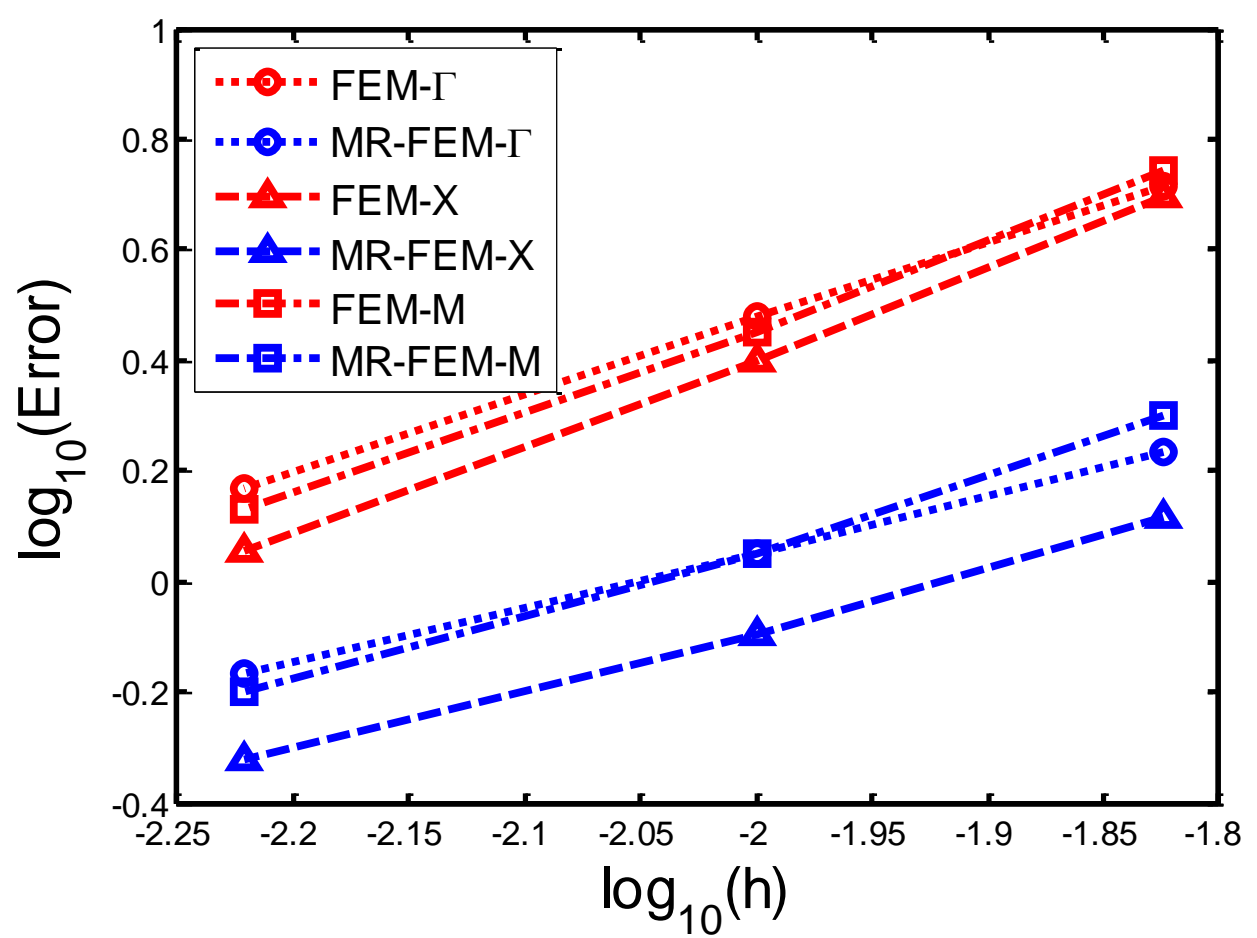

Figure 12: Convergence rate of FEM and MR-FEM 


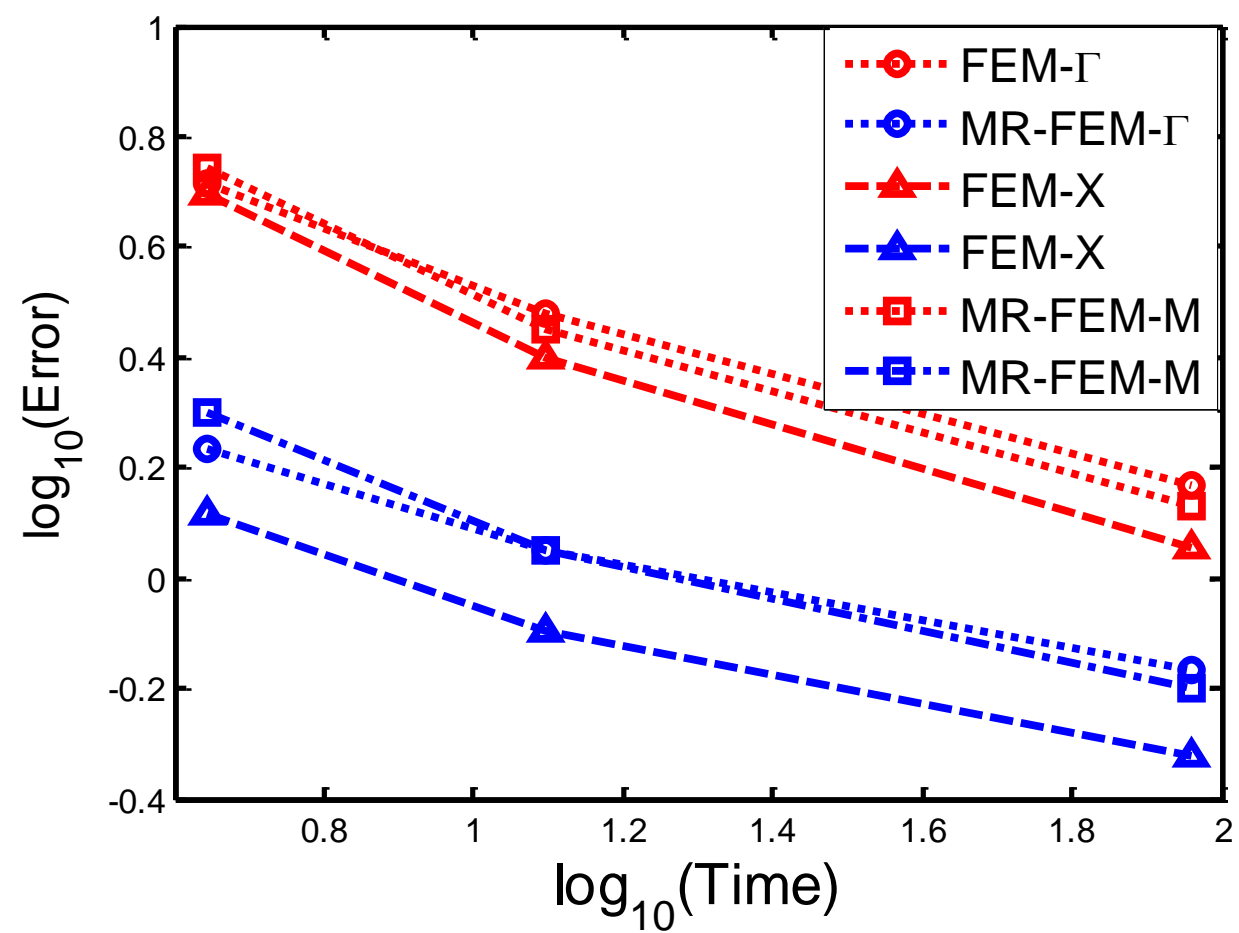

Figure 13: Computational efficiency 


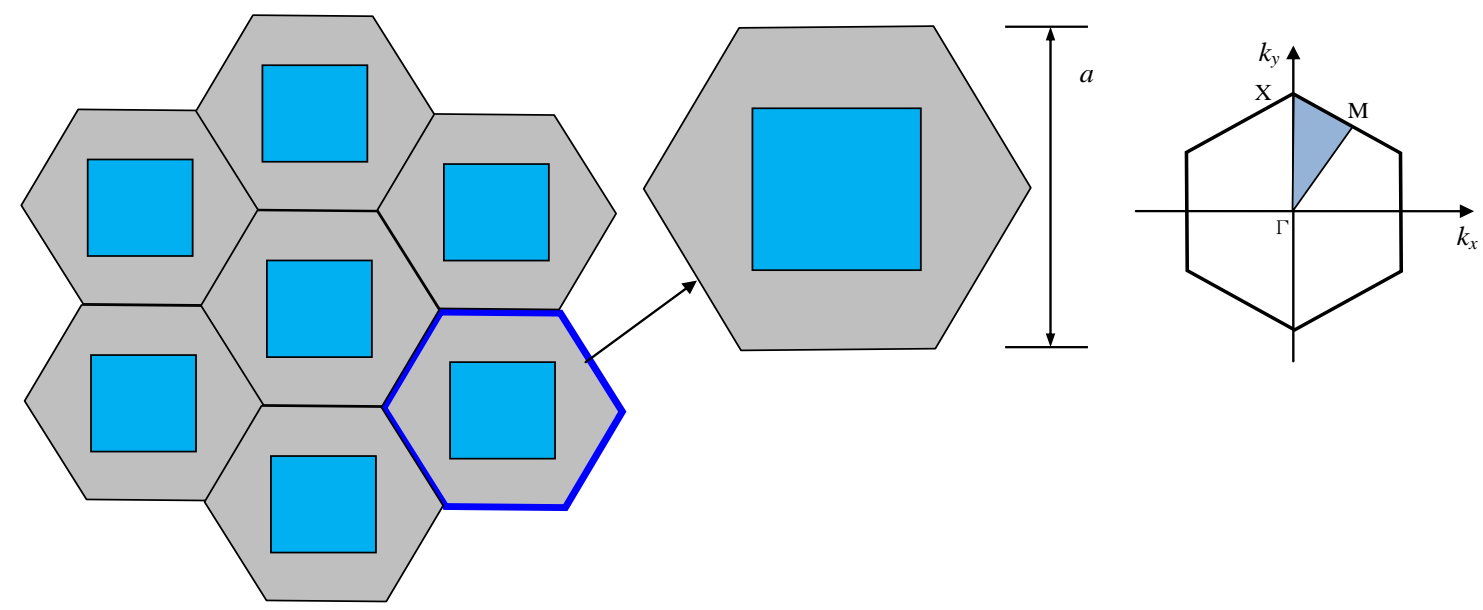

Figure 14: Solid/fluid PCs with triangular lattice 


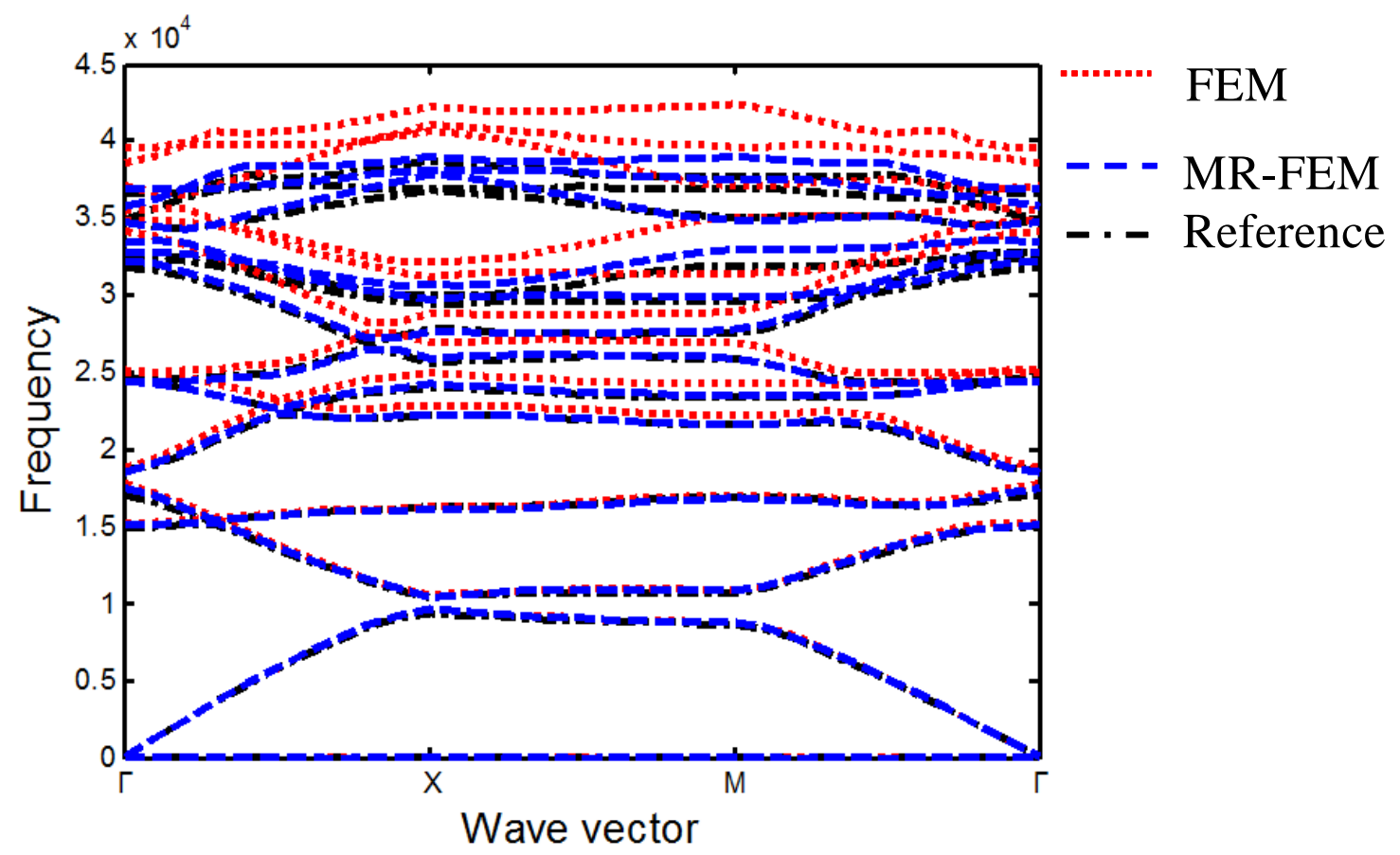

Figure 15: Comparison of band gap using different numerical methods 


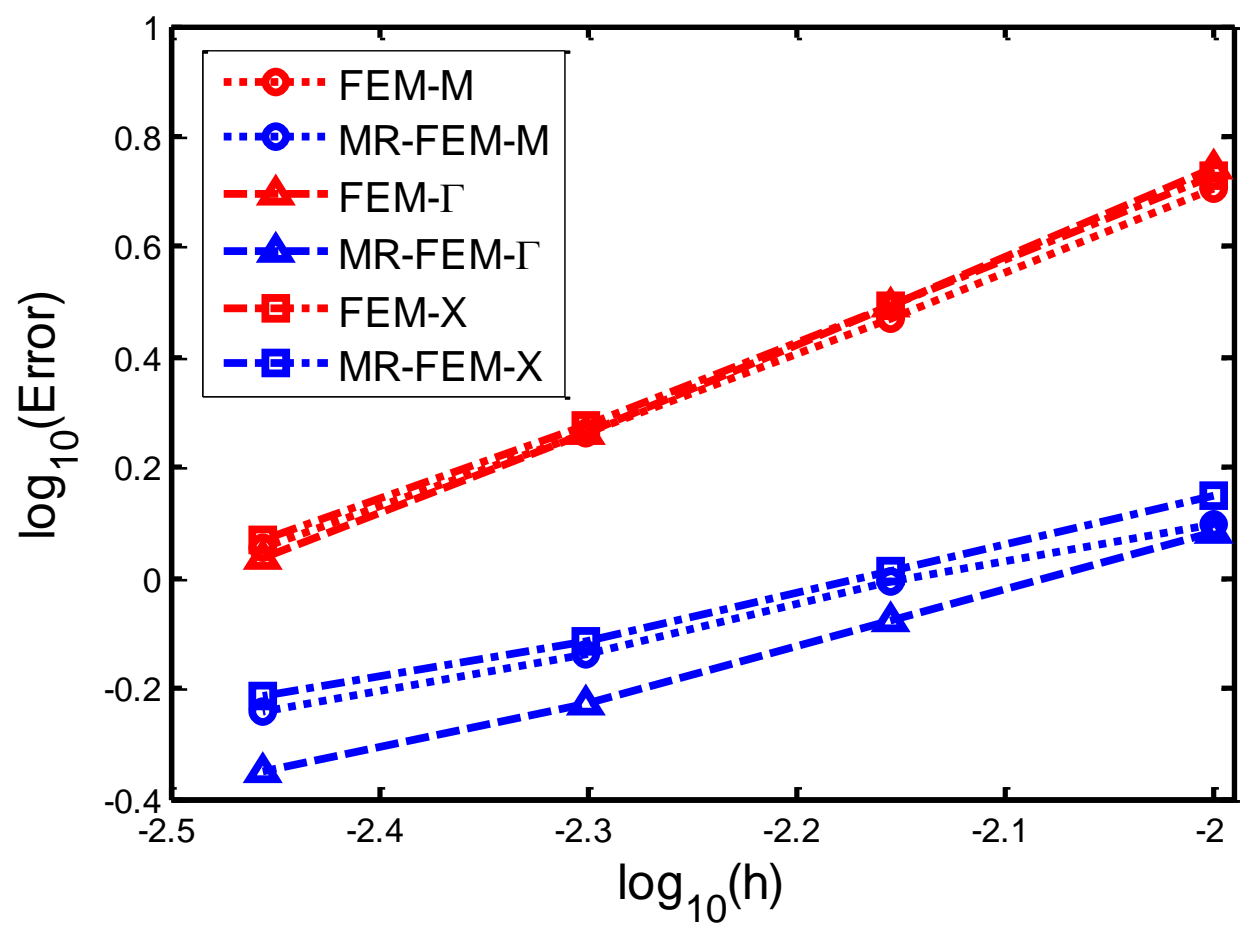

Figure 16: Convergence rate of eigenfrequency for the first fifteen mode shape 


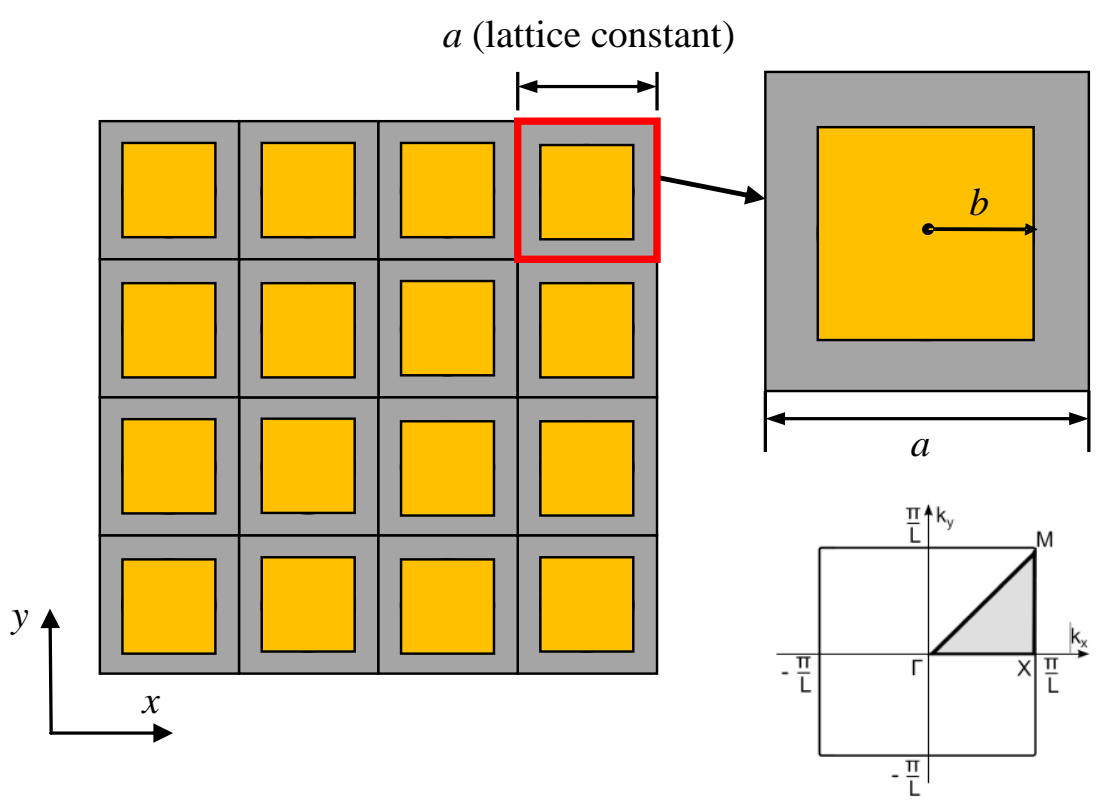

Figure 17: Fluid/solid PCs with square lattice 


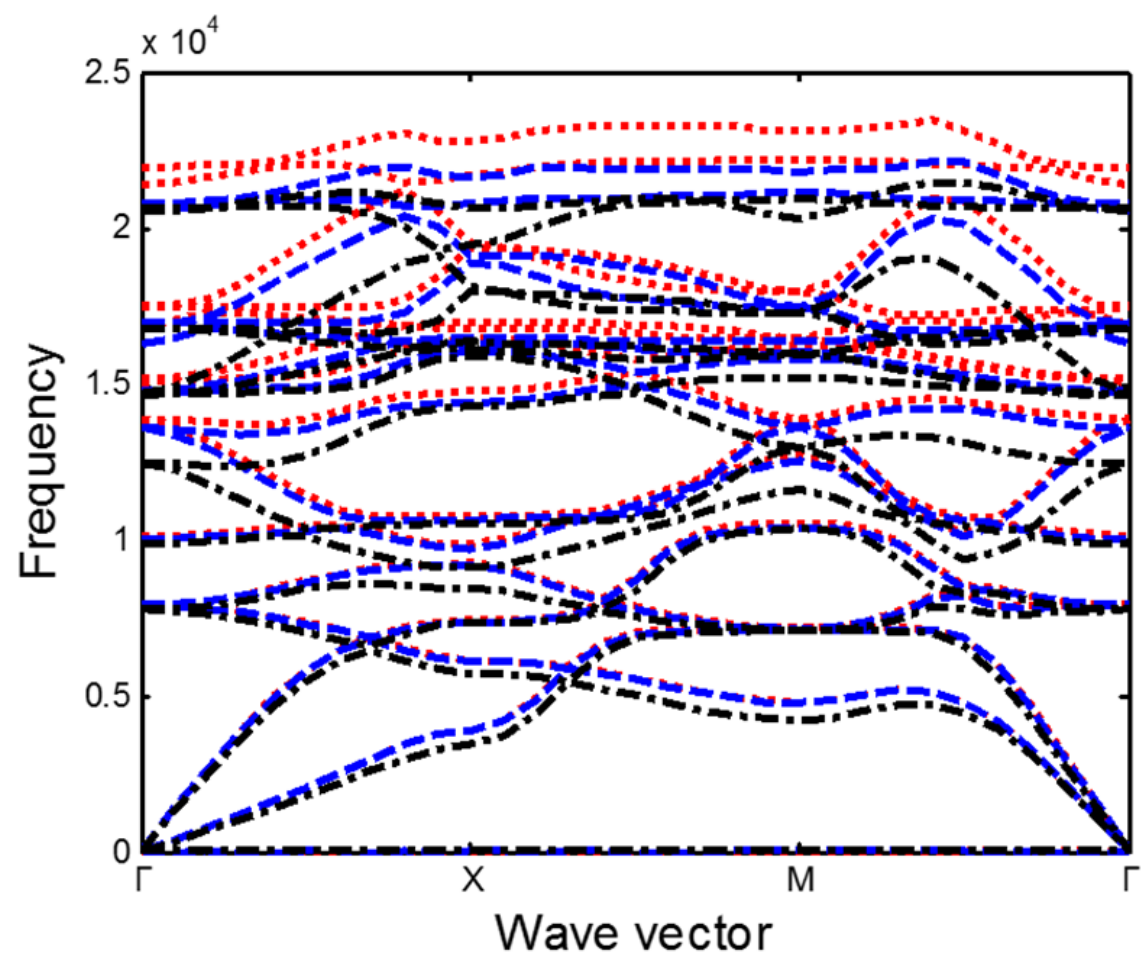

…...... FEM

- - - MR-FEM

-.-. Reference

Figure 18: The numerical results of band gap 


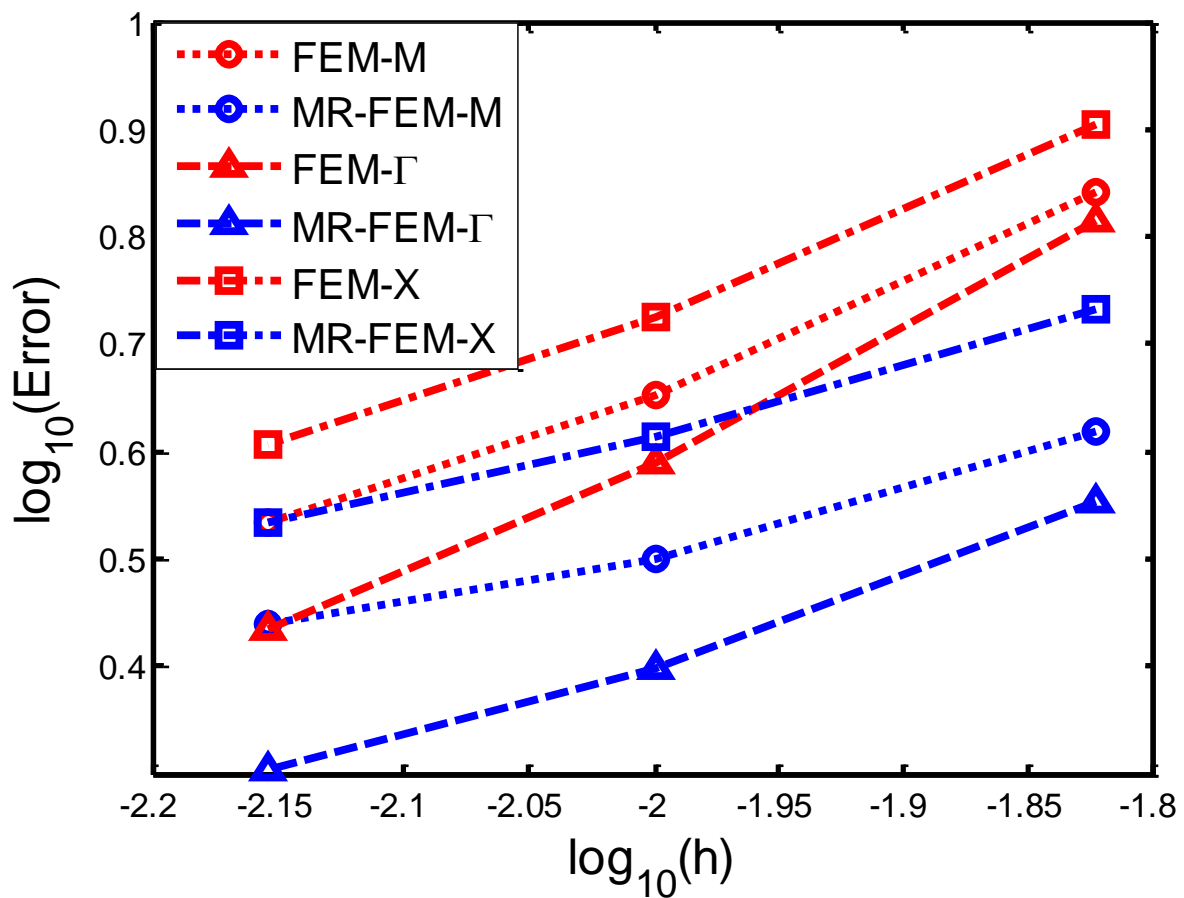

Figure 19: Convergence rate of eigenfrequency for the first fifteen mode shapes 


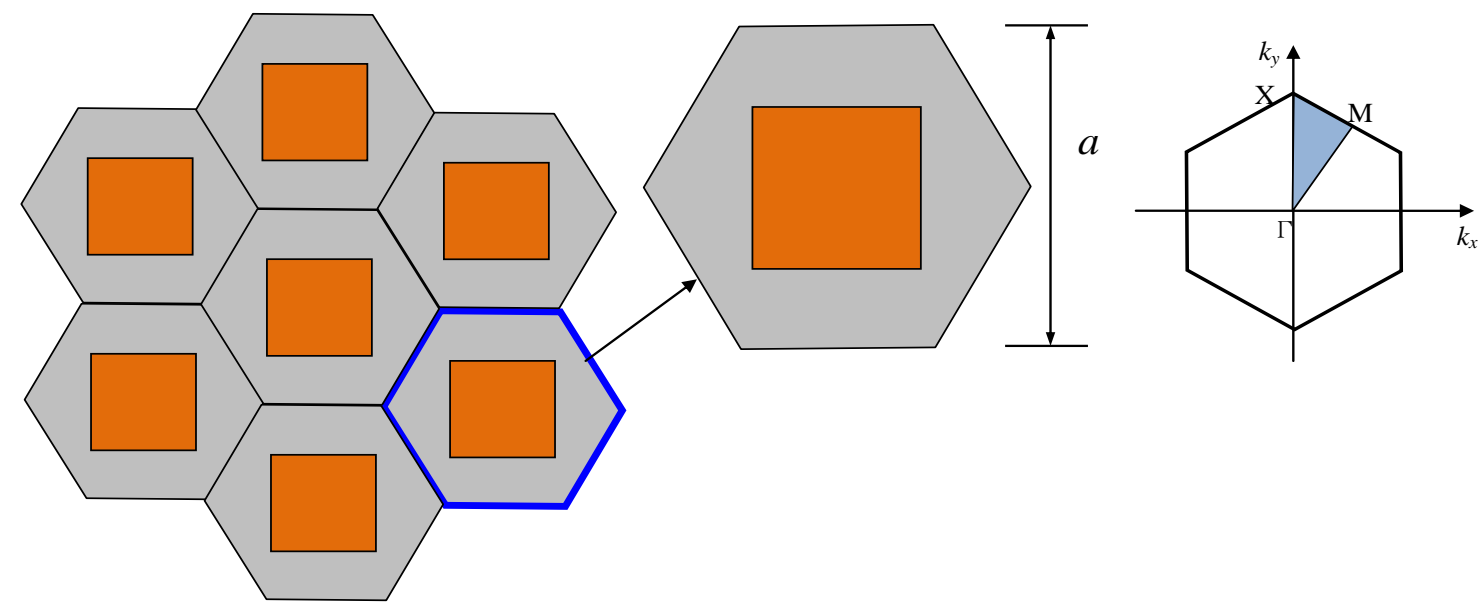

Figure 20: Fluid/solid PCs with triangular lattice 


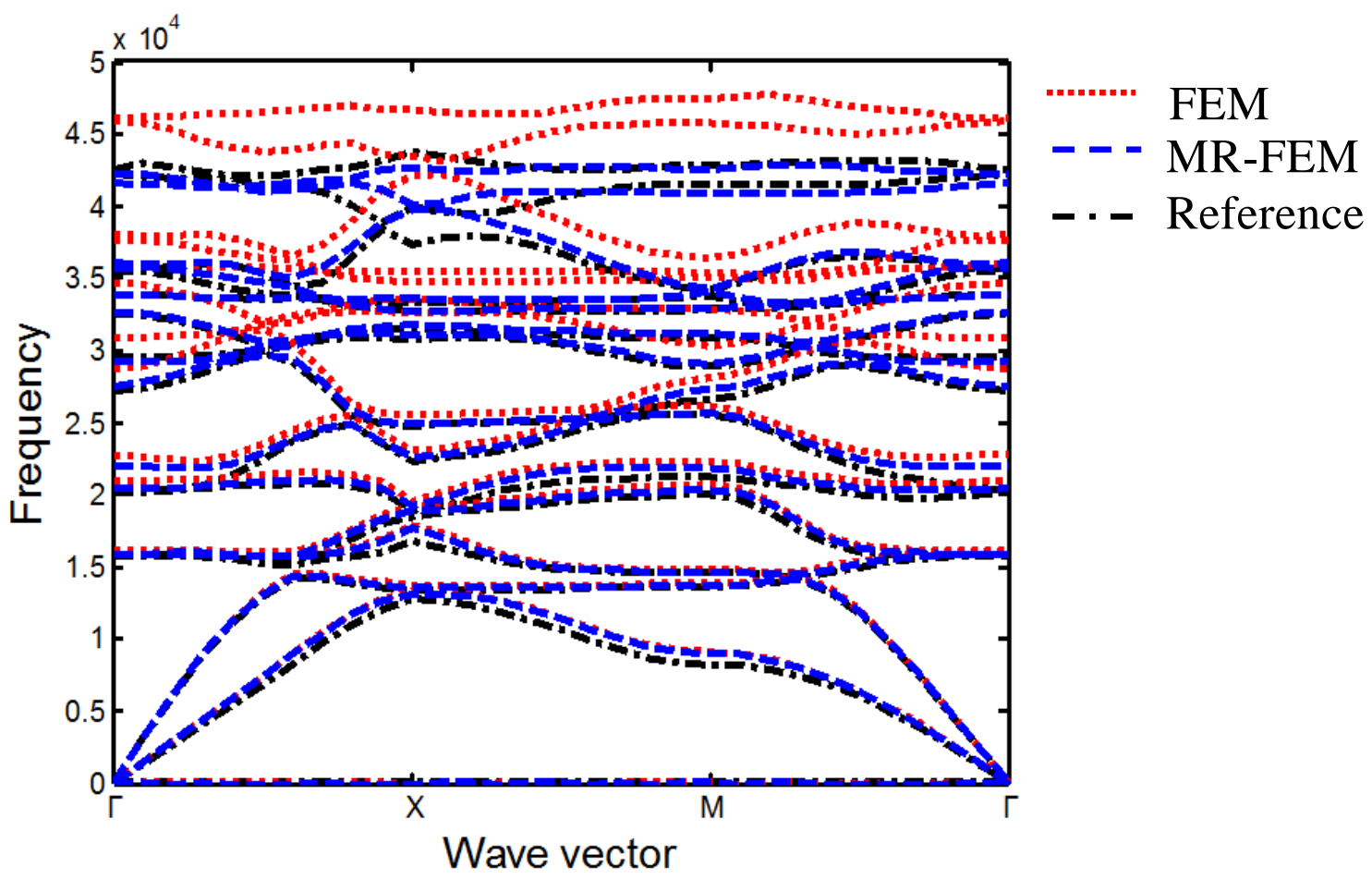

Figure 21: Comparison of band gap using different numerical methods 


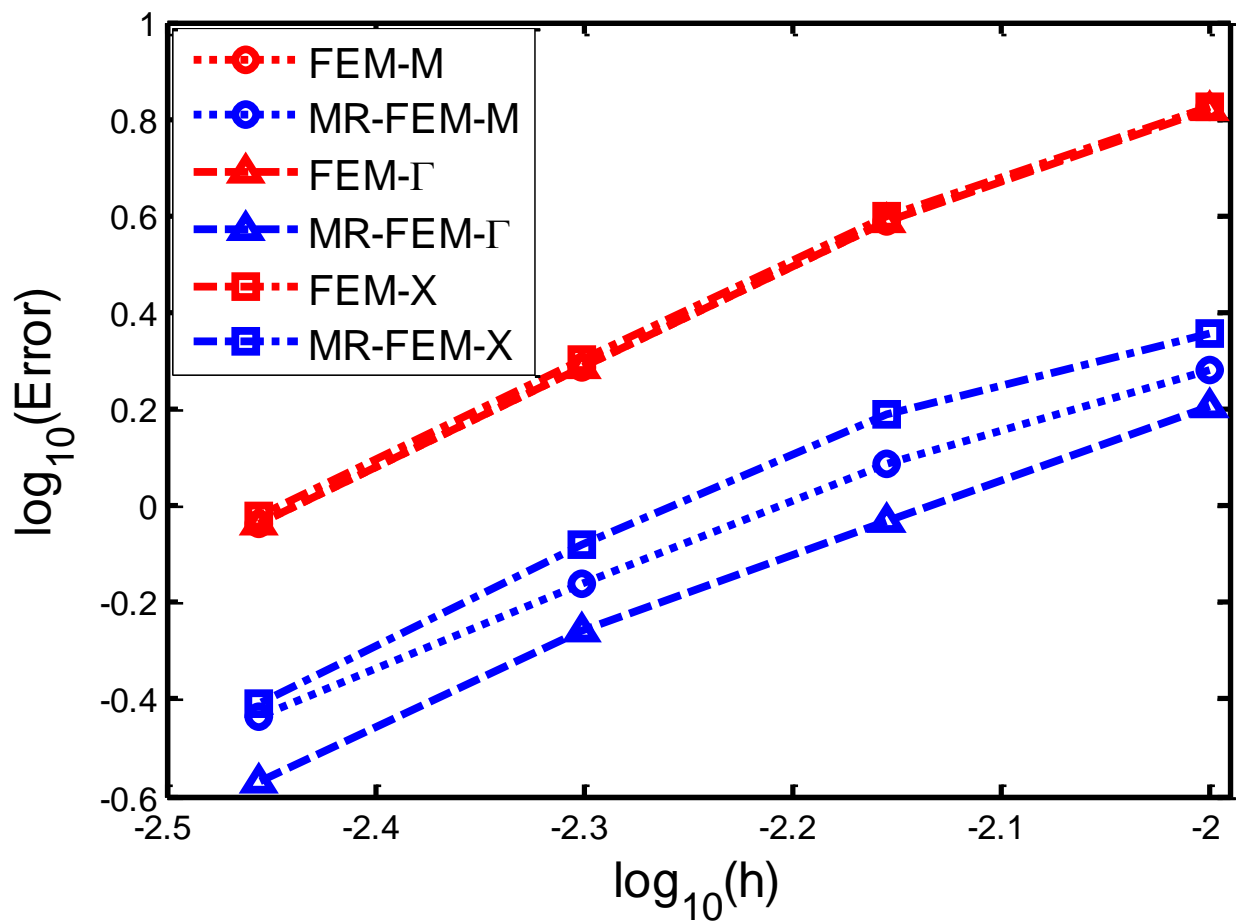

Figure 22: Convergence rate of eigenfrequency for the first fifteen mode shape 


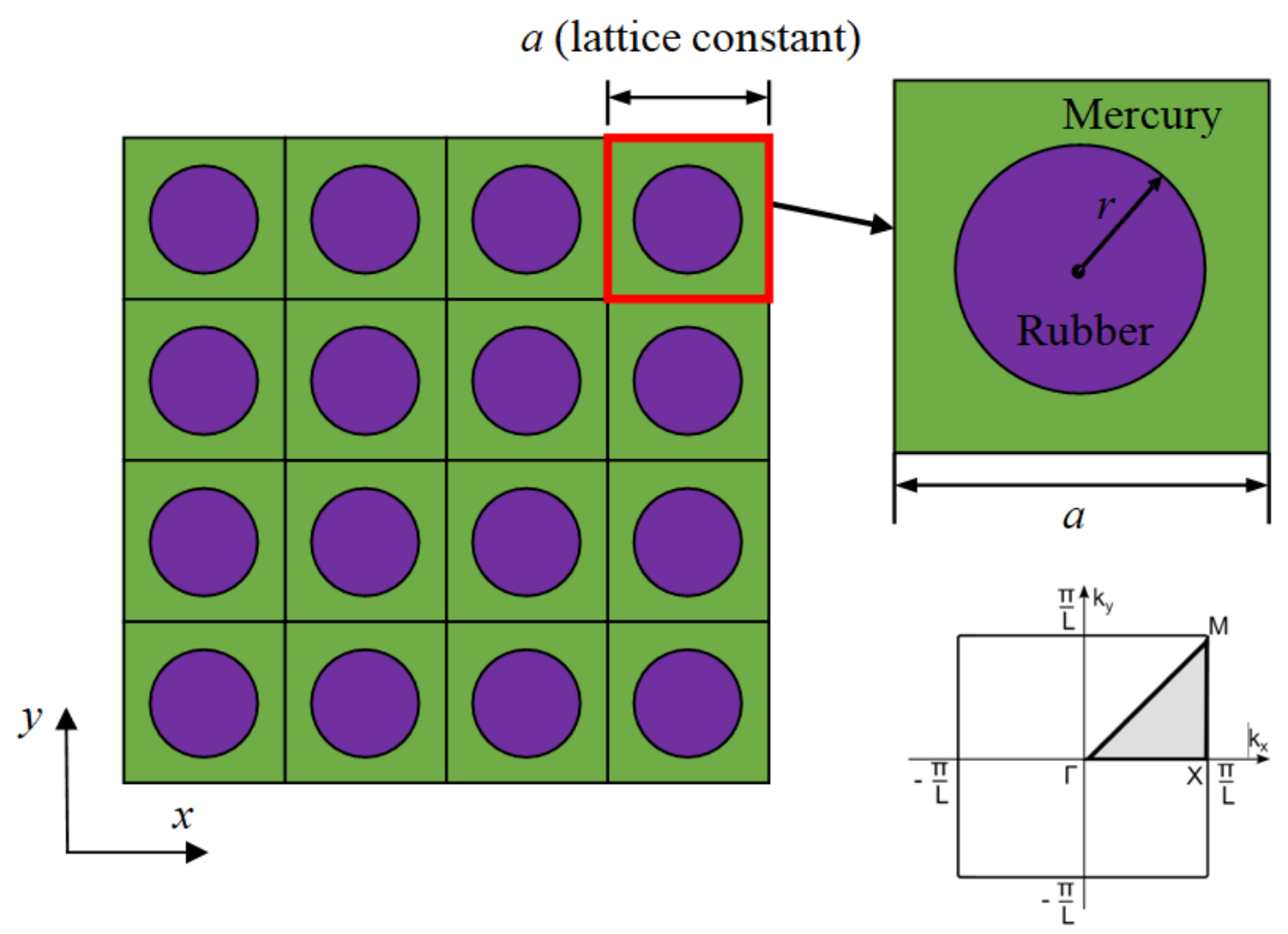

Figure 23: Extreme case (Rubber / Mercury PCs) 


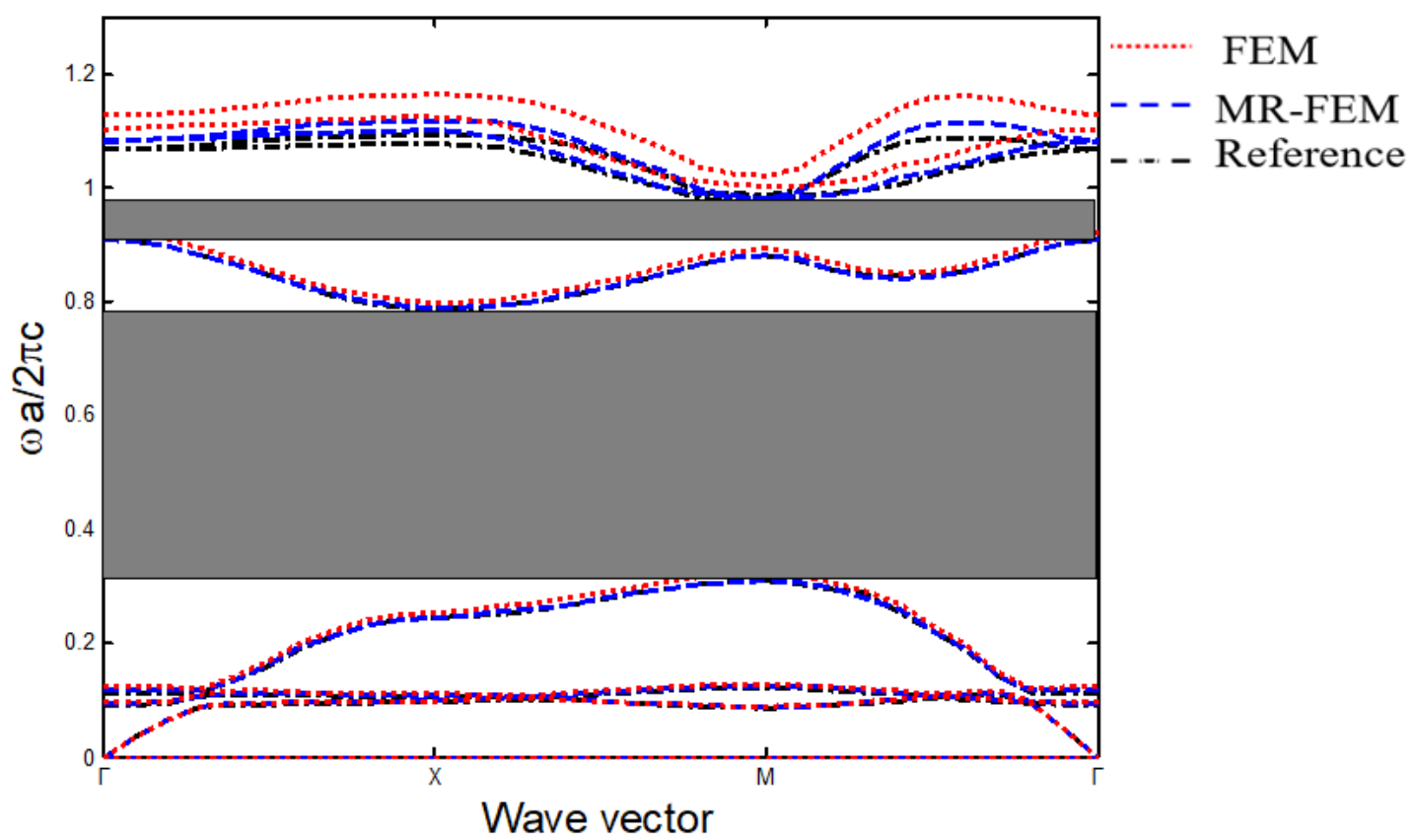

Figure 24: Comparison of band gap using FEM and MR-FEM 
Table 1: Four sets of mesh in fluid/solid PCs with square lattice

\begin{tabular}{cccccc}
\hline \hline Model & $\begin{array}{c}\text { Size of } \\
\text { element }\end{array}$ & $\begin{array}{c}\text { Number of } \\
\text { nodes in solid }\end{array}$ & $\begin{array}{c}\text { Number of } \\
\text { elements in } \\
\text { solid }\end{array}$ & $\begin{array}{c}\text { Number of } \\
\text { nodes in fluid }\end{array}$ & $\begin{array}{c}\text { Number of } \\
\text { elements in } \\
\text { fluid }\end{array}$ \\
1 & 0.025 & 23 & 31 & 55 & 73 \\
2 & 0.015 & 59 & 95 & 109 & 157 \\
3 & 0.010 & 123 & 212 & 194 & 296 \\
4 & 0.0073 & 219 & 391 & 360 & 591 \\
\hline \hline
\end{tabular}

Table 2: Three sets of mesh in Helmholtz resonator PCs with square lattice

\begin{tabular}{cccccc}
\hline \hline Model & $\begin{array}{c}\text { Size of } \\
\text { element }\end{array}$ & $\begin{array}{c}\text { Number of } \\
\text { nodes in solid }\end{array}$ & $\begin{array}{c}\text { Number of } \\
\text { elements in } \\
\text { solid }\end{array}$ & $\begin{array}{c}\text { Number of } \\
\text { nodes in } \\
\text { solid }\end{array}$ & $\begin{array}{c}\text { Number of } \\
\text { elements in } \\
\text { fluid }\end{array}$ \\
1 & 0.015 & 30 & 28 & 82 & 106 \\
2 & 0.010 & 44 & 42 & 152 & 220 \\
3 & 0.006 & 112 & 148 & 306 & 474 \\
\hline \hline
\end{tabular}

Table 3: Four sets of mesh in fluid/solid PCs with triangular lattice

\begin{tabular}{cccccc}
\hline \hline Model & $\begin{array}{c}\text { Average } \\
\text { Size of } \\
\text { element }\end{array}$ & $\begin{array}{c}\text { Number of } \\
\text { nodes in the } \\
\text { solid domain }\end{array}$ & $\begin{array}{c}\text { Number of } \\
\text { elements in the } \\
\text { solid domain }\end{array}$ & $\begin{array}{c}\text { Number of } \\
\text { nodes in the } \\
\text { fluid domain }\end{array}$ & $\begin{array}{c}\text { Number of } \\
\text { elements in the } \\
\text { fluid domain }\end{array}$ \\
2 & 0.010 & 40 & 58 & 96 & 118 \\
3 & 0.007 & 67 & 104 & 152 & 214 \\
4 & 0.005 & 131 & 220 & 244 & 364 \\
\hline \hline
\end{tabular}


Table 4: Three sets of mesh in solid/fluid PCs with square lattice

\begin{tabular}{cccccc}
\hline Model & $\begin{array}{c}\text { Average } \\
\text { Size of } \\
\text { element }\end{array}$ & $\begin{array}{c}\text { Number of } \\
\text { nodes in the } \\
\text { solid domain }\end{array}$ & $\begin{array}{c}\text { Number of } \\
\text { elements in the } \\
\text { solid domain }\end{array}$ & $\begin{array}{c}\text { Number of } \\
\text { nodes in the } \\
\text { fluid domain }\end{array}$ & $\begin{array}{c}\text { Number of } \\
\text { elements in the } \\
\text { fluid domain }\end{array}$ \\
2 & 0.015 & 104 & 140 & 71 & 112 \\
3 & 0.010 & 202 & 304 & 131 & 220 \\
\hline \hline
\end{tabular}

Table 5: Four sets of mesh in solid/fluid PCs with triangular lattice

\begin{tabular}{ccccc}
\hline \hline Model & $\begin{array}{c}\text { Number of nodes } \\
\text { in the solid domain }\end{array}$ & $\begin{array}{c}\text { Number of } \\
\text { elements in the } \\
\text { solid domain }\end{array}$ & $\begin{array}{c}\text { Number of nodes in } \\
\text { the fluid domain }\end{array}$ & $\begin{array}{c}\text { Number of } \\
\text { elements in the } \\
\text { fluid domain }\end{array}$ \\
1 & 96 & 118 & 40 & 58 \\
2 & 152 & 214 & 67 & 104 \\
3 & 244 & 364 & 131 & 220 \\
4 & 447 & 730 & 244 & 430 \\
\hline \hline
\end{tabular}

